\title{
Palaemonidae, Euryrhynchidae, and Sergestidae (Crustacea: Decapoda): Records of native species from the states of Amapá and Pará, Brazil, with maps of geographic distribution
}

\author{
Fabiana Ribeiro Pimentel ${ }^{1}$ and Célio Magalhães ${ }^{2^{*}}$ \\ 1 Instituto Nacional de Pesquisas da Amazônia, Programa de Pós-Graduação em Biologia de Água Doce e Pesca Interior. Av. André Araújo, 2936 - \\ Petrópolis, CEP 69067-375 Manaus, AM, Brazil. \\ 2 Instituto Nacional de Pesquisas da Amazônia, Coordenação de Biodiversidade. Av. André Araújo, 2936 - Petrópolis, CEP 69067-375 Manaus, AM, \\ Brazil. \\ * Corresponding author. E-mail: celiomag@inpa.gov.br
}

\begin{abstract}
A list of the distribution records for 18 species of freshwater shrimps of the families Euryrhynchidae (3 spp.), Palaemonidae (13 spp.) and Sergestidae (2 spp.) from the states of Amapá and Pará are presented. All records are documented by voucher specimens deposited in the three main carcinological collections from northern Brazil: Instituto de Pesquisas Científicas e Tecnológicas do Amapá (Macapá), Instituto Nacional de Pesquisas da Amazônia (Manaus), and Museu Paraense Emílio Goeldi (Belém). Maps of the geographic distribution of these records in the eastern Amazon region are offered, and comments on the zoogeography of the species are made.
\end{abstract}

DOI: $10.15560 / 10.6 .1300$

\section{INTRODUCTION}

The diversity of freshwater shrimps in the Brazilian Amazon basin is well known due to taxonomic reviews by Holthuis (1951, 1952), Omori (1975), Tiefenbacher (1978), and Kensley and Walker (1982). This fauna includes representatives of the families Euryrhynchidae (one genus: Euryrhynchus Miers, 1877), Palaemonidae (three genera: Macrobrachium Bate, 1868; Palaemon Weber, 1795; and Pseudopalaemon Sollaud, 1911), and Sergestidae (one genus: Acetes H. Milne Edwards, 1830). However, species distribution is still poorly known particularly in the eastern Amazon, since collections have been scarce and usually concentrated along larger rivers or in the vicinities of major cities. A few records of freshwater shrimps from Pará were made by Calman (1907), who described Euryrhynchus burchelli, and by Holthuis (1952, 1966), who reported the occurrence of Macrobrachium amazonicum (Heller, 1862), M. nattereri (Heller, 1862), and Palaemonetes carteri Gordon, 1935 from the vicinities of Santarém, State of Pará. Additionally, there are only a few records of freshwater shrimps for the states of Amapá and Pará: Kensley and Walker (1982) recorded Palaemonetes carteri Gordon, 1935, in the Rio Curuá-Una, State of Pará; Ramos-Porto and Coelho (1990) recorded $P$. carteri from Rio Mapiri, Santarém, and listed some species of Macrobrachium in either one or in both states; D'Incao and Martins (2000) recorded Acetes marinus Omori, 1975, and Acetes paraguayensis Hensen, 1919, respectively from Vila Amazonas (Amapá) and the lower Tocantins River (Pará), and Tucusul (= Tucuruí, Pará); Ferreira et al. (2010) recorded Macrobrachium acanthurus (Wiegmann, 1836) from Pará, and Macrobrachium olfersii (Wiegmann, 1836) and Nematopalaemon schmitti (Holthuis, 1950) from both states; and Pileggi et al. (2013) informed a number of records for several species from both states as well.

The occurrence of freshwater shrimps in these two states can also be verified in check lists and faunistic papers (Coelho and Ramos-Porto 1985; Barros and Silva 1997; Ramos-Porto \& Coelho, 1998; Barros and Pimentel 2001; Vieira 2003, 2008; Magalhães and Pereira 2007; Cavalcante et al. 2012) or in several papers dealing with population dynamics (Odinetz Collart 1991, Silva et al. 2005, Bentes et al. 2011, Freire et al. 2012a), growth (Silva et al. 2007), and fisheries (Odinetz Collart 1987; Silva et al. 2002a, b, 2005; Lucena-Frédou et al. 2010; Freire et al. 2012b).

Despite the previous records, there is still much unpublished information about the distribution of freshwater shrimps in carcinological collections of the three main institutions in northern Brazilian. The purpose of this paper is to list the records of freshwater species of Euryrhynchidae, Palaemonidae and Sergestidae from the states of Amapá and Pará housed at the Instituto de Pesquisas Científicas e Tecnológicas do Amapá (Macapá), Instituto Nacional de Pesquisas da Amazônia (Manaus), and Museu Paraense Emílio Goeldi (Belém), so that this information can be useful for taxonomic and zoogeographic studies as well as conservation assessments.

\section{MATERIALS AND Methods}

The records presented herein encompass freshwater shrimps found in the Brazilian states of Amapá (140.276 $\mathrm{km}^{2}$ ) and Pará $\left(1.284 .042 \mathrm{~km}^{2}\right)$, situated between the coordinates $04^{\circ} 20^{\prime} 45^{\prime \prime} \mathrm{N}$ and $09^{\circ} 50^{\prime} 27^{\prime \prime} \mathrm{S}$, and $46^{\circ} 03^{\prime} 18^{\prime \prime}$ 
$\mathrm{W}$ and $58^{\circ} 53^{\prime} 42^{\prime \prime} \mathrm{W}$. The material is deposited in the Instituto de Pesquisas Científicas e Tecnológicas do Amapá (IEPA), Instituto Nacional de Pesquisas da Amazônia (INPA) e Museu Paraense Emílio Goeldi (MPEG). The following abbreviations were used in the text: APEG = Área de Pesquisas Ecológicas do Guamá (Guamá Ecological Research Area); cc = length of cephalotorax (measured dorsally from anterior margin of orbit to posterior margin of cephalotorax); coll. = collector $(\mathrm{s})$; $\mathrm{ct}=$ total length (measured dorsally from tip of rostrum to tip of telson); ECFPn = Estação Científica Ferreira Penna (Ferreira Pena Scientific Station); FLONA = Floresta Nacional (National Forest); juv. = juvenile(s); $\hat{\sigma}=\operatorname{male}(\mathrm{s}) ; \uparrow=$ female $(\mathrm{s})$; $\hat{\sigma}$ immat $=$ immature $\operatorname{male}(\mathrm{s}) ;$ immat. $=$ immature female(s); + ov. $=$ ovigerous female(s); spec. $=\operatorname{specimen}(\mathrm{s})$. Names of landforms are kept in the original language (Portuguese) as written in the labels.

Taxonomic classification follows Martin and Davis (2001), and the taxa are organized alphabetically. Synonymic lists include citations made from the states of Amapá and Pará only.

\section{RESULTS}

Sergestidae Dana, 1852

Acetes H. Milne-Edwards, 1830

\section{Acetes marinus Omori, 1975}

Acetes marinus Omori 1975: 49; Coelho and Ramos-Porto 1985: 408 [in list]; D'Incao 1998: 318 [in list]; D'Incao and Martins 2000: 79, figs. 2a-f; Barros and Pimentel 2001: 19 [in list]; Melo 2003: 404 [map].

Geographic distribution. Suriname and Brazil (Amapá, Pará) (Omori 1975; D’Incao 1998; D’Incao and Martins 2000).

Material examined. AMAPÁ: Lago Piratuba Biological Reserve, Rio Araguari, 16.viii.1992, C. Magalhães, 3 spec., (INPA 750). PARÁ: Baía de Marajó, boca da Vigia, 18.ii.1995, R. Barthem, 51へ̂, 28ㅇ, (MPEG 313); idem, Vigia, 19.ii.1995, idem, 1 ๙ 10 , , (MPEG 314); idem, mouth of Vigia, 10.iii.1995, idem, 93 $\hat{\jmath}, 41$, (MPEG 312); idem,

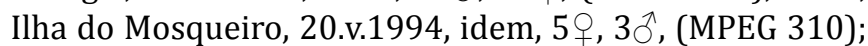
idem, idem, near Ilha Guaiba, 20.v.1994, R. Barthem and R. Aragão, 94 $\hat{\circ}, 65 \bigcirc$, (MPEG 311); idem, Ilha de Mosqueiro, near Ilha Guaíba, 20.v.1994, coll. unknown, 3 spec., (INPA 757); idem, idem, 20.v.1994, R. Barthem and R. Aragão, 8 spec., (INPA 581); idem, mouth of Rio Arari, 05.vii.1984, R. Barthem, 1 spec., (INPA 1098); idem, Ilha Tatuoca, 11.vi.1984, R. Barthem, 1 spec., (INPA 1095); idem, 20.vii.1984, M.L. Carvalho, 15 spec., (INPA 083); Ilha de Marajó, Ponta Fina, Cabo Maguari, 16.v.1984, R. Barthem, 4 spec., (INPA 1241); Igarapé do Paracuri, Icoaraci, 23.xii.1995, M. Mourão Jr., L.M.A. da Silva and R.A.F. Maia, 19, (MPEG 48); mouth of Murucupi, 31.v.1984, R. Barthem, 1 spec., (INPA 763); Belém, Igarapé Sapucajuba (POEMA), 24.i.1995, M. Mourão Jr. and R.A.F. Maia, 19, (MPEG 4); Rio Tocantins, Mangabeira, 08.xi.1980, coll. unknown, 3 spec., (INPA 84); Rio Pará, Ilha das Araras, Curralinho, 25.vii.1984, R. Barthem, 1 spec., (INPA 1096). All records are shown in Figure 1.

\section{Acetes paraguayensis Hansen, 1919}

Acetes paraguayensis Hansen 1919: 46, figs. 8-14; Omori 1975: 54, figs. 8, 23, 24a-k, 25; D’Incao 1998: 318 [in list]; Melo 2003: 406 [map].

Acetes paraguaiensis [sic] - Barros and Pimentel 2001: 19 [in list].

Geographic distribution. Venezuela, Colombia, Peru, Bolivia, Paraguay, Argentina, Brazil (Acre, Amazonas, Pará, Tocantins, Rondônia, Goiás, Mato Grosso) (see Pileggi et al. 2013 for references).

Material examined. PARÁ: Banco das Araras, Curralinho, 25.vii.1984, R. Barthem, 4 spec., (INPA 1096); Ilha do Combú, Acará, 24.x.2001, Adélia, 4 spec., (MPEG 773); Rio Amazonas, Lago Grande de Monte Alegre, 12.viii.1992, C. Alcântara, 62 0 , 45ㅇ, (MPEG 309); Rio Xingu basin, Maroá and Acaraí Rivers, between Senador José Porfírio and Porto de Moz (02 $\left.03^{\prime} 25^{\prime \prime} \mathrm{S} 52^{\circ} 14^{\prime} 56^{\prime \prime} \mathrm{W}\right)$, 08.xi.1994, L. Rapp Py-Daniel et al., 1 spec., (INPA 1092); Rio Tapajós, mouth of Rio Maicá, Santarém, 23.iv.1999,

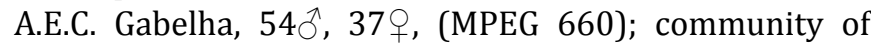
do Guajará, Santarém, 15.v.1999, coll. unkown, 12§, 20오. (MPEG 666); community of Santa Maria do Tapará, Santarém, 21.xi.1999, coll. unknown, 11ð, 11, (MPEG 678); community of São Jorge do Uricurituba, Santarém, 29.xii.1999, M.R. dos Reis, 10 spec., (MPEG 683); Rio Tapajós, mouth of Rio Maicá, Santarém, 23.iv.1999, A.E.C. Gabelha, 1 spec., (INPA 1093); community of Guajará, Santarém, 15.v.1999, R.N. Yuki, 1 spec., (INPA 1094); Rio Tocantins, Igarapé Arapari, Breu Branco, Itupiranga, 24.xi.1981 a 13.vii.1982, coll. unknown, 2 spec., (INPA 225). All records are shown in Figure 1.

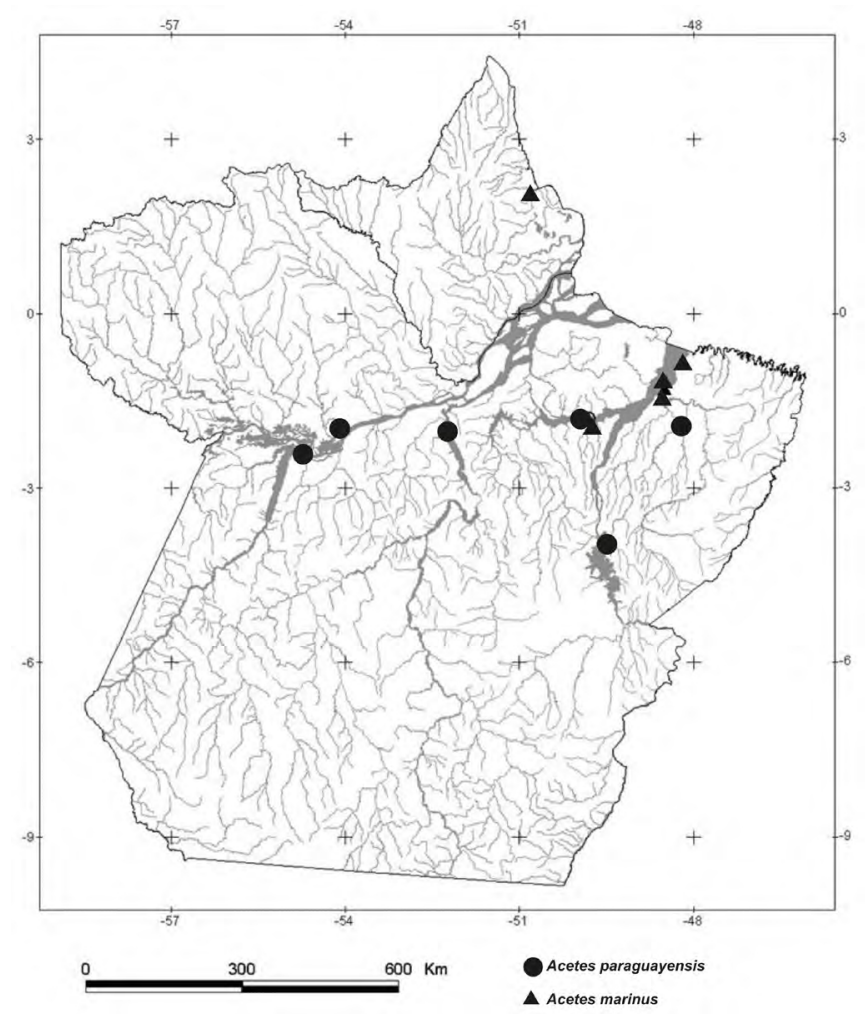

FIGURE 1. Geographic distribution of Acetes marinus Omori, 1975, and A. paraguayensis Hansen, 1919 in the States of Amapá and Pará, Brazil (some symbols may represent more than one record). 
Euryrhynchidae Holthuis, 1950

Euryrhynchus Miers, 1877

Euryrhynchus amazoniensis Tiefenbacher, 1978

Euryrhynchus amazoniensis Tiefenbacher 1978: 183, fig.

2a,b; Kensley and Walker 1982: 2, figs. 1-2; Magalhães and Pereira 2007: 14 [in list].

Euryrrhynchus [sic] amazoniensis - Vieira 2003: 59, fig.

Geographic distribution. Peru, Venezuela, Brazil (Amazonas, Pará) (Tiefenbacher 1978; Kensley and Walker, 1982; Pereira 1991; Delgado et al. 1997; GarcíaDávila and Magalhães 2004).

Material examined. AMAPÁ: Rio Araguari, unnamed igarapé near Cachoeira da Capivara, 20.viii.1992, C. Magalhães et al., 11§̂, 22ᄋ, 4 juv., (INPA 1081); Serra do Navio, Igarapé Piçarra, 22.iii.1991, V. Py-Daniel et al., 1ో̂, (INPA 1014).

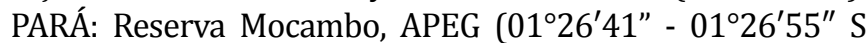
$48^{\circ} 25^{\prime} 44^{\prime \prime}-48^{\circ} 25^{\prime} 34^{\prime \prime}$ W), Belém, 01.viii.1998, F.R. Pimentel and R.N. Yuki, 1 + , 3 9immat., (MPEG 600); idem, 15.ix.1998, idem, $10 \hat{O}, 1$, 1 immat., (MPEG 611); idem, 15.xii.1998, idem, 1 immat., (MPEG 603); idem, 21.i.1999, F.R. Pimentel and R.M. dos Santos, 19, (MPEG 614); idem, 24.ii.1999, F.R. Pimentel and J.O. Dias, $2 \widehat{\jmath}, 1$, , (MPEG 618); idem, 19.iii.1999, idem, 1ㅇ, 1 ov., (MPEG 621); idem, 22.iv.1999, F.R. Pimentel and R.M. dos Santos, 4 ते, (MPEG 624); idem, 21.v.1999, F.R. Pimentel and J.O. Dias, 19, (MPEG 627); idem, 18.vi.1999, idem, 1ㅇ, 1 immat., (MPEG 630); idem, 21.vii.1999, idem, 4ð̂, 2 , , (MPEG 633); idem, 19.viii.1999, F.R. Pimentel and R.M. dos Santos, 3ð̂, 1 ov., (MPEG 636); idem, 30.ix.1999, F.R. Pimentel and J.O. Dias, $1 \hat{\jmath}, 1$,, 2 juv., (MPEG 639); idem, 19.i.2000, idem, 29, (MPEG 648); Lago Jacundá, Alterdo-Chão, Santarém, 16.ii.2000, C.R. García-Dávila, 40, 8 , 3 ov., (INPA 2009); Lago Verde, Alter-do-Chão, Santarém, 18.ii.2000, C.R. García-Dávila, 2 + , (INPA 2035); Lago Caravari, Alter-do-Chão, Santarém, 16.ii.2000, C.R. García-Dávila, 4옹, (INPA 2012); left bank of Rio Tapajós near mouth of Rio Cupari, 27.x.1991, C. Magalhães and L. Rapp Py-Daniel, 19, (INPA 1205); Rio Xingu, Ilha do Forno, Altamira, 18.xii.2000, R. Souza and Dionísio, 11§, 7q, (MPEG 713); idem, Altamira, 16.xii.2000, R. Souza and Masinho, $2 \hat{\jmath}$, (MPEG 711); idem, Igarapé do Ipixuna, 22.x.1992, coll. unknown, 40, 6우, (INPA 1151); idem, 30.ix.1990, coll. unknown, 1 , (INPA 1165);

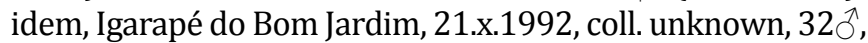
23오. (INPA 1167); idem, Lago do Poção, Igarapé do Poção, 11.x.1992, V. Py-Daniel et al., 10̂, 1 †immat., (INPA 1169);

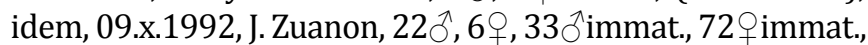
(INPA 1172); idem, Lago do São Sebastião, 18.x.1992, coll. unknown, $10 \AA$, 1q, 12§immat., 9 immat., (INPA 1173); Rio Trombetas Basin, Igarapé Borges, tributary to Rio Mapuera, 27.iv.1985, V. Py-Daniel et al., 42 spec., (INPA 265); Lago Tapagem, right bank of Rio Trombetas, 11.x.1985, C. Magalhães, 13 spec., (INPA 337); left bank of Tapajós River, between Buburé and São Luis, 22/23.x.1991, C. Magalhães and L. Moraes, 69 $\hat{\circ}$, 49으, (INPA 1191); Rio Jamanxim, Ilha da Terra Preta, 20.x.1991, Raimundo, 1+, (INPA 1198). All records are shown in Figure 2.

\section{Euryrhynchus burchelli Calman, 1907}

Euryrhynchus Burchelli Calman 1907: 297, figs. 2-8.

Euryrhynchus burchelli - Holthuis 1951: 9, est. 2, figs. g-m;

Tiefenbacher 1978: 185, fig. 2c,d; Rodríguez 1981: 46 [in list]; Ramos-Porto and Coelho 1998: 330 [in list]; Barros and Pimentel 2001: 20 [in list]; Vieira 2003: 60, fig.; Melo 2003: 324 [map]; De Grave 2007: 194.

Geographic distribution. Brazil (Amazonas, Pará) (Holthuis 1951; 1966; Kensley and Walker 1982).

Material examined. AMAPÁ: Rio Amapá Grande, Cachoeira Grande, small Igarapé upstream from the fall, Amapá, 26.viii.1992, C. Magalhães and Roberval,

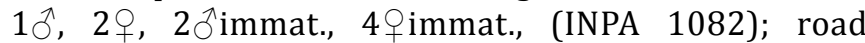
Macapá-Fazendinha, Poço Museu Costa Lima, Macapá, 17.v.1990, S. Borges, 2ð, 1ㅇ, (IEPA 00027); J.K. road (IEPA - Centro Zoobotânico), Poço Amazonas, Km 10, Fazendinha district, Macapá, 18.xi.1995, H.P. Belo, 1q, (IEPA 00050). PARÁ: Igarapé Bacuri, Santarém-Novo $\left(00^{\circ} 55^{\prime} 45^{\prime \prime} \mathrm{S} 47^{\circ} 23^{\prime} 50^{\prime \prime} \mathrm{W}\right)$, 26.iv.1998, F.R. Pimentel and R.N. Yuki, $7 \hat{\jmath}, 2 \bigcirc, 4{ }_{+}$ov., (MPEG 565); Reserva Mocambo, APEG, (012 $26^{\prime} 41^{\prime \prime}-01^{\circ} 26^{\prime} 55^{\prime \prime}$ S, 48 $25^{\prime} 44^{\prime \prime}$ - 48 $25^{\prime} 34^{\prime \prime}$ W), Belém, 17.ix.1997, M.P. de Barros, F.R. Pimentel, S.S.B. da Silva and R.N. Bittencourt, 1 ov., (MPEG 450); bica da APEG, Belém, 17.ix.1997, idem, 1 spec., (MPEG 457); Reserva Mocambo, APEG,

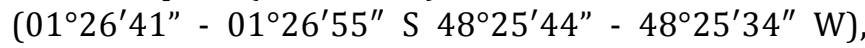
Belém, 09.i.1998, F.R. Pimentel and R.A.F. Maia, 19ð̄, $10 \bigcirc, 4$ ov., (MPEG 529); of MPEG research campus, viii.1998, R.N. Yuki, 3 3 , 2 ㅇ, 2 ov., (MPEG 585); Reserva

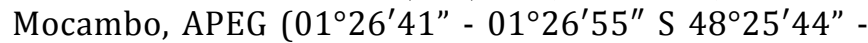
$48^{\circ} 25^{\prime} 34^{\prime \prime}$ W), Belém, 14.vii.1998, F.R. Pimentel and R.N. Yuki, 1ð, 2 ㅇ, 1 ov., (MPEG 597); idem, 01.viii.1998, idem, 17ठ, 3ㅇ, (MPEG 602); idem, 15.ix.1998, idem, 7ð̂, 4ㅇ, 3 oov., (MPEG 599); idem, 20.x.1998, F.R. Pimentel and D. Guimarães, 5 $\hat{0}, 2$, (MPEG 605); idem, 18.xi.1998, F.R. Pimentel and R.N. Yuki, $6 \hat{\jmath}, 12+, 4+$ ov.,

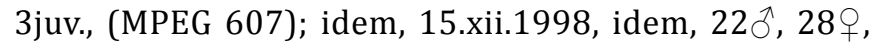
(MPEG 610); idem, 21.i.1999, F.R. Pimentel and R.M. dos Santos, 8ㅅ, 8ㅇ, 1 ov., (MPEG 613); idem, 24.ii.1999, F.R. Pimentel and J.O. Dias, 60, 4 , (MPEG 617); idem, 19.iii.1999, idem, $11 \hat{\delta}, 8$, 8 orv., (MPEG 620); idem, 22.iv.1999, F.R. Pimentel and R.M. dos Santos, $7 \hat{\jmath}, 6$, 2 ov., (MPEG 623); idem, 21.v.1999, F.R. Pimentel and J.O. Dias, $5 \hat{\jmath}, 4 \bigcirc, 2 \hat{\jmath}$ immat., 5 immat., 1 imov., (MPEG 626); idem, 18.vi.1999, idem, 11ऽ, $20 \circ, 19$ ov., (MPEG 629); idem, 21.vii.1999, idem, $8 \hat{\jmath}, 4+1$, 1 ov., (MPEG 632); idem, 19.viii.1999, F.R. Pimentel and R.M. dos Santos, 5ð̂, 5ㅇ, (MPEG 635); idem, 30.ix.1999, F.R.

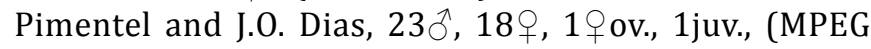
638); idem, 21.x.1999, idem, 110̄, 15 9 , 6 ov., (MPEG

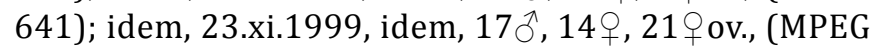
643); idem, 22.xii.1999, idem, 20, 16 , 15 ov., (MPEG 645); idem, 19.i.2000, idem, $9 \hat{\jmath}, 5$ + 2 ov., (MPEG 647); idem, 24.ii.2000, idem, $7 \hat{\jmath}, 5$ q 2 qov., (MPEG 650);

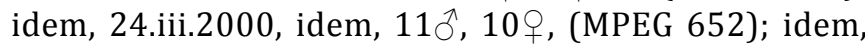

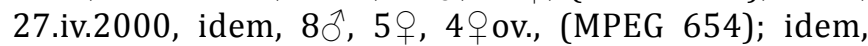
25.v.2000, idem, $7 \hat{\jmath}, 4$, (MPEG 656); Vila do Conde,

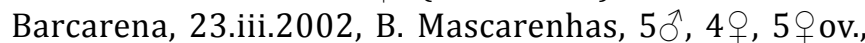
(MPEG 740); Vila Arienga, Igarapé Arienga, Barcarena, 26.iii.2002, B. Mascarenhas, 9 spec., (MPEG 745); Vila do Conde, Linhão, Barcarena, 25.iii.2002, B. Mascarenhas et al., 2 spec., (MPEG 752); Reserva Genética Felipe, Monte Dourado, 14.iv.2002, B. Mascarenhas et al., 1 spec., (MPEG 748); ECFPn, FLONA de Caxiuanã, Rio Curuá,

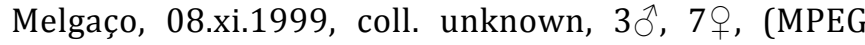


716); idem, Igarapé Poraquequara, Melgaço, 07.xi.1999, coll. unknown, 5ð̄, 3 ㅇ, (MPEG 721); idem, 06.xi.1999, coll. unknown, 10今, 5 , (MPEG 724); idem, 10.xi.1999, coll. unknown, 1ㅇ, (MPEG 725); idem, mouth of Curuá River, Melgaço, xi.1999, coll. unknown, 1 \% , (MPEG 732); idem, Melgaço, 02.vii.2000, Ferreira and Adélia, 2 spec., (MPEG 756); idem, Rio Curuá, Melgaço, 07.vii.2000, B. Mascarenhas, 1 @immat., (MPEG 764); idem, Rio Curuá, Melgaço, 18.iii.2001, coll. unknown, 2 qov., (MPEG 766); Lago Jacundá, Alter-do-Chão, Santarém, 16.ii.2000, C.R. García-Dávila, $2 \hat{\jmath}, 1+7+$ ov., (INPA 1108); Lago Caravari, Alter-do-Chão, Santarém, 16.ii.2000, C.R. García-Dávila, 69, (INPA 1111); Igarapé do Irurama, Alter-do-Chão, Santarém, 17.ii.2000, C.R. García-Dávila, 10̃, (INPA 1115); headwaters of Lago Preto, Alter-do-Chão, Santarém, 17.ii.2000, C.R. García-Dávila, $5 \hat{\jmath}, 7$, 1 ov., (INPA 1123); headwaters of Lago Verde, Alter-do-Chão, Santarém, 18.ii.2000, C.R. García-Dávila, 20, 6, 11 ov., (INPA 1132); Lago Verde, Alter-do-Chão, Santarém, 18.ii.2000, C.R. García-Dávila, 3, (INPA 1136); Lago Minitiapina, Alter-do-Chão, Santarém, 19.ii.2000, C.R. García-Dávila, 1ㅇ, (INPA 1127); Lago Iruçanga, Alter-do-Chão, Santarém, 21.ii.2000, C.R. García-Dávila, $2 \widehat{\jmath}, 2$, (INPA 1144); Lago Jacaré, Alter-do-Chão, Santarém, 21.ii.2000, C.R. GarcíaDávila, $2 \hat{O}, 1 q, 1 q$ ov., (INPA 1145); Lago das Mangueiras, Alter-do-Chão, Santarém, 21.ii.2000, C.R. García-Dávila, 1오. (INPA 1148); left bank of Rio Tapajós near mouth of Rio Cupari, 27.x.1991, C. Magalhães and L. Rapp Py-Daniel, 1§ิ, 2ᄋ, (INPA 1202); left bank of Rio Tapajós near mouth of Rio Cupari, 26.x.1991, C. Magalhães and J. Zuanon, 3 immat., (INPA 1203). All records are shown in Figure 2.

\section{Euryrhynchus wrzesniowskii Miers, 1877}

Euryrhynchus wrzesniowskii Miers 1877: 662, est. 67, figs. 2; Holthuis 1951: 5, pl. 1, figs. a-m; Kensley and Walker 1982: 3; Ramos-Porto and Coelho 1998: 330 [in list]; Barros and Pimentel 2001: 20 [in list]; Melo 2003: 326 [map]; Magalhães and Pereira 2007: 14 [in list]; Vieira 2008: 69 [in list].

Euryrhnchus [sic] wrzesniowskii - Vieira 2003: 60, fig.

Geographic distribution. Guyana, Suriname, French Guiana, Brazil (Amazonas, Pará) (Holthuis 1948; 1951; Tiefenbacher 1978; Kensley and Walker 1982; Coelho and Ramos-Porto 1998).

Material examined. PARÁ: Rio Trombetas basin, Igarapé do Patauá, left bank tributary to Rio Mapuera, 09.x.1985, C. Magalhães, $1 \widehat{\jmath}, 1$ + $2{ }^{\Uparrow}$ immat., 2 immat., (INPA 332); idem, igarapé do Km 10 of BR-163 road, near Cachoeira Porteira, 07.x.1985, idem, $7 \hat{\jmath}, 4+, 5 \widehat{\jmath}$ immat., 2 immat., 1 ov., (INPA 333); idem, 15.iv.1985, E. Ferreira and M. Jegu, $3 \bigcirc, 2$ ㅇ, 5 ov., (INPA 1238); idem, igarapé on the left bank of Rio Coxi-Pacoré, 61 Km da BR-163, 16.x.1985, C. Magalhães, $1 \hat{\jmath}, 2$, 1 îmmat., 1 immat., (INPA 1080); Lago Tapagem, right bank of Rio Trombetas, 11.x.1985, idem, 2 ov., (INPA 338); unnamed igarapé tributary to Rio Mapuera, 04 a 09.vi.1986, V. Py-Daniel et al., 3ત, 2 ㅇ, 2 immat., (INPA 448); Rio Trombetas, Igarapé Gaivota,

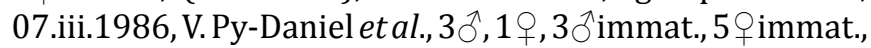
3 ov., (INPA 1079); ECFPn, FLONA de Caxiuanã, Igarapé Poraquequara, Melgaço, 07.xi.1999, coll. unknown, 12 spec., (MPEG 720); idem, 06.xi.1999, coll. unknown, $8{ }^{\lambda}$, $2 \bigcirc$, (MPEG 723); Santarém/Curuá-Una road, Igarapé do Guaraná, 14.xii.1977, I. Vieira et al., 1ठ̉. (INPA 098); Lago Jacundá, Alter-do-Chão, Santarém, 16.ii.2000, C.R. GarcíaDávila, $2 \hat{\jmath}, 2 \circ, 1{ }^{\lambda}$ immat., 1 ov., (INPA 1110); headwaters of Lago Preto, Alter-do-Chão, Santarém, 17.ii.2000, C.R. GarcíaDávila, $4 \widehat{O}, 1$, 4 ov., (INPA 1122); Igarapé São Sebastião, Alter-do-Chão, Santarém, 17.ii.2000, C.R. García-Dávila, 12 $\overbrace{}^{1}$, $13 \circ, 12$ ov., (INPA 1118). All records are shown in Figure 2.

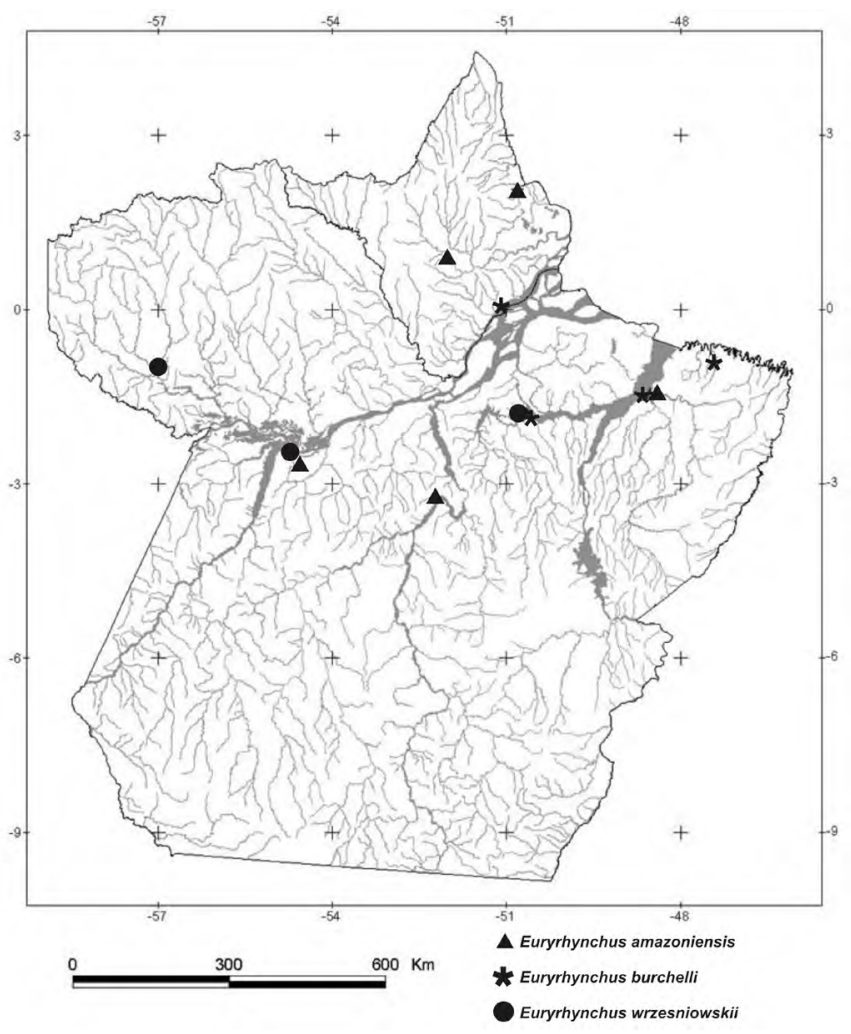

FIGURE 2. Geographic distribution of Euryrhynchus amazoniensis Tiefenbacher, 1978, E. burchelli Calman, 1907, and E. wrzesniowskii Miers, 1877 in the States of Amapá and Pará, Brazil (some symbols may represent more than one record).

\section{Palaemonidae Rafinesque, 1815}

Macrobrachium Bate, 1868

\section{Macrobrachium acanthurus (Wiegmann,1836)}

Palaemon acanthurus Wiegmann, 1836: 150.

Macrobrachium acanthurus - Holthuis 1952: 45, est. 8-9, figs. a-b; Ramos-Porto and Coelho 1998: 331 [in list]; Barros and Pimentel 2001: 20 [in list]; Melo 2003: 338 [map].

Geographic distribution. USA (Florida, Louisiana, Mississipi, Texas, Georgia, Carolina do Norte), Caribbean islands, Gulf of México, Venezuela, Colômbia, Brazil (from Pará to Rio Grande do Sul) (Holthuis 1952; Bond-Buckup 1989; Delgado et al. 1997; Coelho and Ramos-Porto 1998; Bowles et al. 2000).

Material examined. AMAPÁ: Vila de Sucuriju, Amapá, 18.iv.2001, I.M. Vieira and O.M. Costa, 1ठ̄. (IEPA 00260). PARÁ: Ilha Nova ("Rato"), São Caetano de Odivelas, 17.iv.2000, L.A. Souza and N. Bittencourt, 10̂, (MPEG 734); Furo Taici, Bragança, 08.ii.1997, M.P. de Barros and R.V. do Espírito Santo, 5へ, 1, (MPEG 92); idem, 26.iv.1997, M.P. de Barros, $9 \hat{\jmath}, 7$, $5{ }^{3}$ immat., 3 immat., 
(MPEG 171); idem, 15.vi.1997, M.P. de Barros and L.M.A. da Silva, 3 $\hat{\jmath}, 3$, 3 îmmat., 14 juv., (MPEG 265); idem, 12.vi.1997, L.M.A. da Silva and R.V. do Espírito Santo, $3 \widehat{\jmath}$, 4ㅇ, 1 îmmat., (MPEG 280); idem, 09.viii.1997, M.P. de

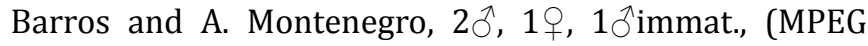
429); idem, 27.ix.1997, idem, $3 \circ$, (MPEG 467); Cachoeira do Arari, mouth of Rio Goiapi, Ilha de Marajó, 08.ix.1990, I.

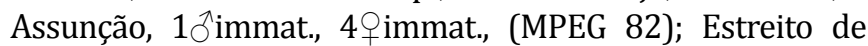

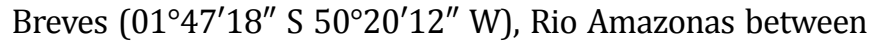
Breves and Curralinho, 17.xi.1994, L. Rapp Py-Daniel et al., 1 ov., (INPA 1089); Rio Urucurrioicaia ( $01^{\circ} 35^{\prime} \mathrm{S} 52^{\circ} 11^{\prime} \mathrm{W}$ ), Rio Amazonas between Porto de Moz and Gurupá, 11.xi.1994,

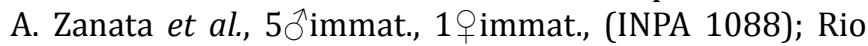
Tapajós $\left(02^{\circ} 23^{\prime} 17^{\prime \prime}\right.$ S $54^{\circ} 42^{\prime} 42^{\prime \prime}$ W), between Cabeça d'Onça and Santarém, 01.xi.1994, O.T. Oyakawa et al., $2 \precsim$ immat., (INPA 1090). All records are shown in Figure 3.

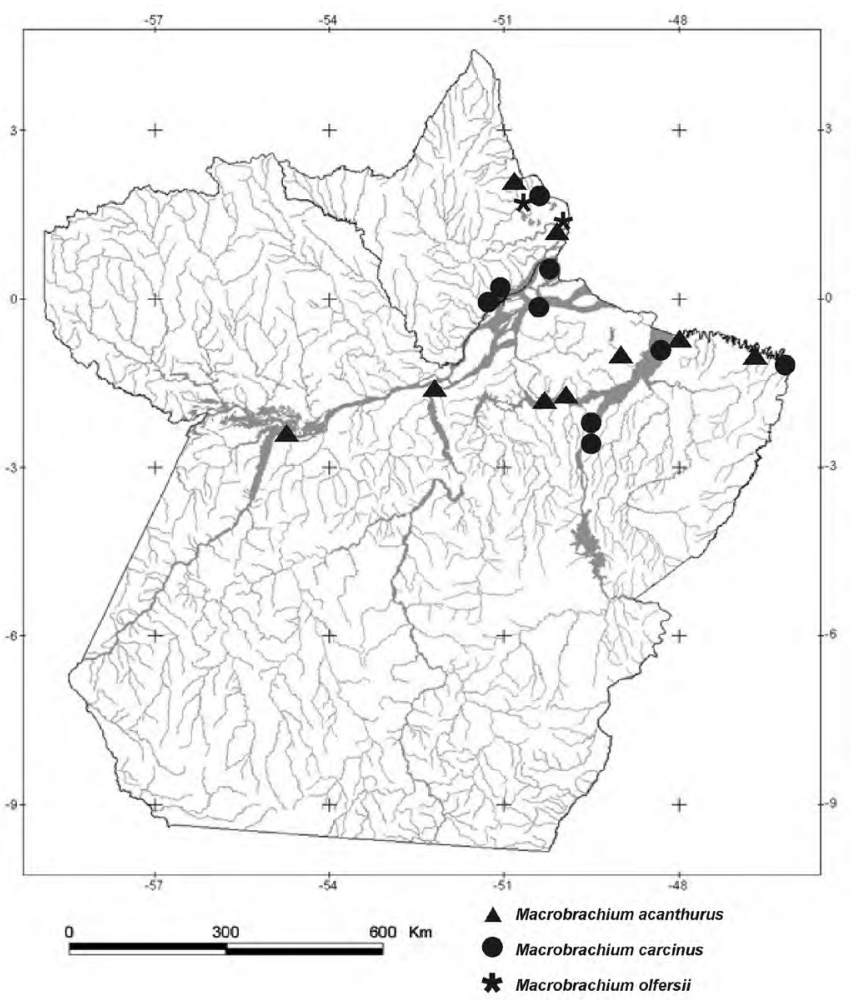

FIgURE 3. Geographic distribution of Macrobrachium acanthurus (Wiegmann,1836), M. carcinus (Linnaeus, 1758), and $M$. olfersii (Wiegmann, 1836) in the States of Amapá and Pará, Brazil (some symbols may represent more than one record).

\section{Macrobrachium amazonicum (Heller, 1862)}

Palaemon amazonicus Heller 1862: 418, pl. 2, fig. 45.

Macrobrachium amazonicum - Holthuis 1952: 18, pl. 2, figs. a-h; Holthuis 1966: 2; Coelho and Ramos-Porto 1985: 408 [in list]; Ramos-Porto and Coelho 1998: 331 [in list]; Barros and Pimentel 2001: 20 [in list]; Vieira 2003: 60, fig.; Melo 2003: 340; Magalhães and Pereira 2007: 14 [in list]; Pileggi and Mantelatto 2010: 196 [in list]; Cavalcante et al. 2012 [in list].

Geographic distribution. Venezuela, Colombia, Suriname, Guyana, Peru, Bolivia, Paraguay, Argentina, Brazil (Acre, Roraima, Rondônia, Amapá, Amazonas, Pará, Maranhão, Piauí, Ceará, Rio Grande do Norte, Paraíba, Pernambuco, Bahia, Tocantins, Goiás, Mato Grosso, Mato Grosso do Sul, Minas Gerais, São Paulo, Paraná) (see Pileggi et al. 2013 for references).

Material examined. AMAPÁ: Rio Amapá Grande, Cachoeira Grande, Amapá, 26.viii.1992, C. Magalhães, 1 , (INPA 1061); Lago Pracuúba, Amapá, vi.1984, M.A.S. Lima, 15 spec., (IEPA 00007); Arquipélago do Bailique, mouth of Igarapé Franco Grande, Amapá, 24.viii.1995, J. Cardoso, N. Farripas and I.M. Vieira, 105 $\hat{\jmath}, 280+, 46 q$ ov., (IEPA 00042); Arquipélago do Bailique, mouth of Igarapé do Veadinho, Amapá, 25.viii.1995, J. Cardoso, N. Farripas and I.M. Vieira, 37 spec., (IEPA 00043); Arquipélago do Bailique, mouth of Igarapé Veado Grande, Amapá, 26. viii.1995, I.M. Vieira, N. Farripas and J. Cardoso, 21 spec., (IEPA 00044); Arquipélago do Bailique, Furo do Igarapé do Veadinho, Amapá, 29.viii.1995, I.M. Vieira, N. Farripas and J. Cardoso, 34 spec., (IEPA 00045); Arquipélago do Bailique, mouth of Rio Faustino, Amapá, 31.viii.1995, L. Ramos, J.C. Cardoso and N. Farripas, $1+$, (IEPA 00049); right bank of Rio Sucuriju, Igarapé Piratuba, Amapá, 26.ix.1996, I.M. Vieira, H.P. Belo and O. Moraes Costa, 1 spec., (IEPA 00116); Distrito de Sucuriju, Amapá, iii.1998, O.M. Costa, I.M. Vieira and A.C. de Souza, 1 spec., (IEPA 00161); Distrito de Sucuriju, Amapá, 23.iii.1998, 0.M. Costa, I.M. Vieira and A.C. de Souza, 1 spec., (IEPA 00167); Arquipélago do Bailique, mouth of Igarapé do Franco Grande, Amapá, xi.1997, I.M. Vieira and Raimundinho, 11 spec., (IEPA 00198); Arquipélago do Bailique, Amapá, 21 a 26.vi.2000, I.M. Vieira, O.A. Alencar and O.M. Costa, 5 spec., (IEPA 00220); Vila de Sucuriju, Amapá, 18.iv.2001, I.M. Vieira and O.M. Costa, 1q, (IEPA 00261); Rio Araguari, Vista Alegre, Lago Piratuba Biological Reserve, 15.viii.1992, C. Magalhães, $6 \hat{\sigma}, 7 \nmid, 1 \delta$ immat., 3 immat., (INPA 1059); Maracá Ecological Station, Jipioca, Igarapé do Cobra, 22.xi.1995, H.P. Belo, J. Cardoso and I.M. Vieira, 2 spec., (IEPA 00073); Fazendinha, between Estaleiro do SENAVA and Cascalheira, Macapá, 25.v.1990, J.C.S. Oliveira, 7 spec., (IEPA 00012); left bank of Rio Amazonas between Fazendinha and Cascalheira, Macapá, 06.v.1990, J.C.S.Oliveira, 11 spec., (IEPA 00020); left bank of Rio Amazonas between Fazendinha and Cascalheira, Macapá, 25.v.1990, J.C.S. Oliveira, 13 spec., (IEPA 00021); Cascalheira Mirim, Distrito da Fazendinha, Macapá, 23. iv.1996, J. Cardoso, A.Merces, H.P. Belo, O.M. Costa, J.N. Mendes and I.M. Vieira, 1 spec., (IEPA 00132); Fazendinha, Macapá, 14.vi.1996, J. Cardoso, A. Merces, H.P. Belo, O.M. Costa, J.N. Mendes and I.M. Vieira, 1 spec., (IEPA 00133); Pau Furado, Santana, 12.v.1981, col. unknown, 1§, 4ㅇ, 2juv., (IEPA 00004); Pau Furado, Santana, v.1981, col. unknown, 1 spec., (IEPA 00005); Ilhas Rosa-Chagas, Igarapé da Fortaleza, Santana, 13.i.1998, col. unknown, $13 \AA, 3+$, 8 ov., (IEPA 00153); Ilhas Rosa-Chagas, Igarapé da Fortaleza, Santana, 20.i.1998, col. unknown, 29 $\hat{\jmath}, 10$, 28 ov., (IEPA 00154); Ilha de Santana, Santana, 14.iv.2000, I.M. Vieira, O.A. Alencar, O.M. Costa, J. Cardoso and A.C. de Souza, 20 spec., (IEPA 00211); Ilha de Santana, Santana, 14 e 15.iv.2000, I.M. Vieira, O.A. Alencar, O.M. Costa, J. Cardoso and A.C. de Souza, 15 spec., (IEPA 00213); Ilha de Santana, Santana, 14-15.iv.2000, I.M. Vieira, O.M. Costa and O.A. Alencar, 1 q , (IEPA 00249). PARÁ: Rio Maniva, Ilha do Pará, Afuá, 19-24.viii.1998, O.A. Alencar, O.M. Costa and A.C. de Souza, 15 $\jmath^{\lambda}, 27$, (IEPA 00188); Rio Maniva, Ilha do Pará, Afuá, 18-20.vi.1999, A.C. de Souza, I.M. Vieira, O.A. Alencar and O.M. Costa, 26 spec., (IEPA 00194); Rio Maniva, Ilha do 
Pará, Afuá, 16.xii.1999, 0.A. Alencar and O.M. Costa, 11 spec., (IEPA 00196); Ilha do Pará, Igarapé do Anta, Afuá, 13-14.iv.2000, I.M. Vieira, O.A. Alencar, O.M. Costa, J. Cardoso and A.C. de Souza, 21 spec., (IEPA 00210); Ilha do Pará, Igarapé do Anta, Afuá, 14 e 15.iv.2000, I.M. Vieira, O.A. Alencar, O.M. Costa, J. Cardoso and A.C. de Souza, 18 spec., (IEPA 00212); Ilha do Pará, Igarapé do Anta, Afuá, 11.xii.2000, I.M. Vieira, O.A. Alencar and O.M. Costa, 1 spec., (IEPA 00236); Ilha do Pará, mouth of Igarapé do Anta, Afuá, 13-14.iv.2000, I.M. Vieira, O.A. Alencar, J. Cardoso, O.M. Costa and A.C. de Souza, $1+$, (IEPA 00247); Ponta Seca entre Vigia e Colares $\left(00^{\circ} 51^{\prime} 31^{\prime \prime} \mathrm{S} 48^{\circ} 08^{\prime} 31^{\prime \prime} \mathrm{W}\right), 19$. xi.1994, J. Roberto, $3{ }^{\circ}, 1$, , (MPEG 69); Vigia $\left(00^{\circ} 51^{\prime} 31^{\prime \prime} \mathrm{S}\right.$

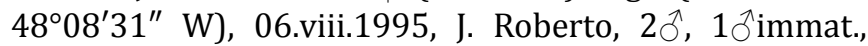
1 immat., 1juv., (MPEG 70); Igarapé Anuerá, Vigia $\left(00^{\circ} 51^{\prime} 31^{\prime \prime} \mathrm{S} 48^{\circ} 08^{\prime} 31^{\prime \prime} \mathrm{W}\right), 22 . x .1995$, R.A.F. Maia and M. Mourão Jr., $4 \hat{O}, 19,2$ ov., (MPEG 5); Igarapé Anuerá, Vigia $\left(00^{\circ} 51^{\prime} 31^{\prime \prime} \mathrm{S} 48^{\circ} 08^{\prime} 31^{\prime \prime} \mathrm{W}\right), 19 . i i .1995$, R.A.F. Maia and M. Mourão Jr., 13ð, 2 ,, 6 ov., (MPEG 17); idem, 06.viii.1995,

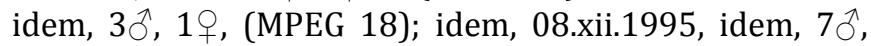
19 ov., (MPEG 21); Furo Taici $\left(00^{\circ} 58^{\prime} 216^{\prime \prime} \mathrm{S} 46^{\circ} 44^{\prime} 370^{\prime \prime}\right.$ W), Bragança, 08.ii.1997, M.P. de Barros and R.V. do Espírito Santo, 14 $\delta^{\circ}$ 10, (MPEG 93); Furo Taici (0058'216" S 4644'370" W), Bragança, 26.iv.1997, M.P. de Barros and R. Pinheiro, 18 ${ }^{\lambda}, 30$, , (MPEG 170); Augusto Corrêa bridge, Bragança, 26.iv.1997, D. Guimarães, 12へ,

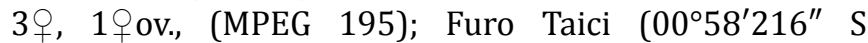
4644'370" W), Bragança, 25.iv.1997, M.P. de Barros and L.M.A. da Silva, 7ð, 3ㅇ, 13juv., (MPEG 198); Furo Taici $\left(00^{\circ} 58^{\prime} 216^{\prime \prime} \mathrm{S} 46^{\circ} 44^{\prime} 370^{\prime \prime} \mathrm{W}\right)$, Bragança, 15.vi.1997, M.P. de Barros and L.M.A. da Silva, 36 $\bigcirc^{\lambda}, 23 \bigcirc$, 10juv., (MPEG 264); idem, 12.vi.1997, L.M.A. da Silva e R.V.do Espírito Santo, $11 \hat{\jmath}, 7$, , 8juv., (MPEG 281); Furo Taici $\left(00^{\circ} 58^{\prime} 216^{\prime \prime}\right.$ S 464' $370^{\prime \prime}$ W), Bragança, 09.viii.1997, M.P. de Barros and A. Montenegro, 11 $0^{\circ}, 7$, (MPEG 428); Furo Taici $\left(00^{\circ} 58^{\prime} 216^{\prime \prime} \mathrm{S} 46^{\circ} 44^{\prime} 370^{\prime \prime} \mathrm{W}\right)$, Bragança, 27.ix.1997, M.P. de Barros and A. Montenegro, 12 $0^{1}, 8 \circ$, (MPEG 466); Furo Taici $\left(00^{\circ} 58^{\prime} 216^{\prime \prime} \mathrm{S} 46^{\circ} 44^{\prime} 370^{\prime \prime} \mathrm{W}\right)$, Bragança, 28.ix.1997, A. Montenegro, 19, (MPEG 472); Inajá, Igarapé Vilemar, Primavera, 07.ix.1987, B. Mascarenhas et al., 1 9,1 đimmat., 1 iqimmat., (MPEG 759); Rio Maracanã, Mangue do Guilherme, Santarém-Novo $\left(00^{\circ} 55^{\prime} 45^{\prime \prime}\right.$ S 4723'50" W), 25.iv.1998, F.R. Pimentel and R.N. Yuki, $3 \jmath^{\lambda}, 49,3$ ov., (MPEG 567); Cachoeira do Arari $\left(01^{\circ} 00^{\prime} 52^{\prime \prime} \mathrm{S} 48^{\circ} 57^{\prime} 49^{\prime \prime}\right.$ W), mouth of Rio Goiapi, Ilha de Marajó, 28.x.1990, I. Assunção, 2 îmmat., 8 Oimmat., (MPEG 78); idem, 08.

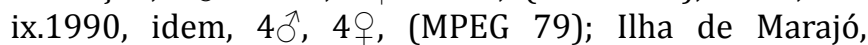
Cachoeira do Arari, mouth of Rio Goiapi, 08.ix.1990, I.

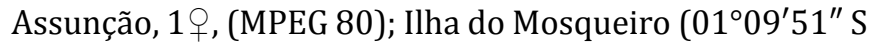
$\left.48^{\circ} 28^{\prime} 20^{\prime \prime} \mathrm{W}\right)$, Igarapé Cajueiro, 07.vii.1994, 1, $3{ }^{\lambda}$,

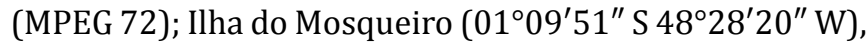
06.ix.1998, B. Silva, $1 \delta^{\top}, 6$ \% 1 1 ov., (MPEG 581); Igarapé do Paracuri, Icoaraci $\left(01^{\circ} 18^{\prime} 07^{\prime \prime} \mathrm{S} 48^{\circ} 29^{\prime} 06^{\prime \prime} \mathrm{W}\right), 23 . x i i .1995$, R.A.F. Maia, L.M.A. da Silva and M. Mourão Jr., 56 $\hat{\jmath}, 32$, 8 ov., (MPEG 34); idem, 07.xi.1995, idem, 16 $\overbrace{}^{\circ}, 19$, (MPEG 36); idem, 24.viii.1995, R.A.F. Maia and L.A. Filgueiras, 11ð, 7 , , (MPEG 37); idem, 09.vii.1995, R.A.F. Maia and M. Mourão Jr., 12 $\oint^{\circ}, 5$, (MPEG 39); idem, 24.vi.1995, idem, 3 immat., 7 Pimmat., (MPEG 40); idem, 06.xi.1994, M. Mourão Jr. and M. Soares, $7 \hat{\jmath}, 3$, 2 ov., 2juv., (MPEG 43); idem, 18.xii.1994, M. Mourão Jr. and R.A.F. Maia, 4ㅇ, 8ภ, 3 Pimmat., (MPEG 55); idem, 21.xii.1994, idem, 6 îmmat.,
3 immat., (MPEG 57); idem, 23.xii.1994, idem, 6ภimmat., (MPEG 60); Igarapé do Paracuri, Icoaraci $\left(01^{\circ} 18^{\prime} 07^{\prime \prime} \mathrm{S}\right.$ 48²9'06" W), 12.iv.1995, R.A.F. Maia and M. Mourão Jr., 3 Pimmat., 6juv., (MPEG 88); Igarapé do Paracuri, Icoaraci (0118'07" S 48²9'06" W), 10.ix.1995, idem, 1ð, (MPEG 334); idem, 26.v.1995, idem, 2へ, 3 , (MPEG 337); Rio Maguari, Ilha do Outeiro, 02.vii.2000, F.R. Pimentel, 1 , 3 3 immat., 2 †immat., (MPEG 693); Igarapé Porto da Seringa, Ananindeua (01 $21^{\prime} 57^{\prime \prime}$ S 48 $22^{\prime} 21^{\prime \prime}$ W), 02. xi.1984, P. Sá and J. Ivan, $3{ }^{3}$ immat., 22 immat., (MPEG 126); Igarapé Sapucajuba (POEMA), Belém, 24.xii.1995, R.A.F. Maia and M. Mourão Jr., $9 ð, 1+, 2+$ ov., 2juv., (MPEG 1); Igarapé Sapucajuba (POEMA), Belém, 13.x.1995, R.A.F. Maia and M. Mourão Jr., 12 $\hat{\jmath}, 9$, (MPEG 8); idem, 31. iii.1995, R.A.F. Maia and I.B. da Silva, $3 \widehat{\partial}$, (MPEG 9); idem,

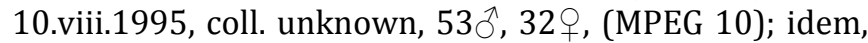
27.xii.1995, R.A.F. Maia and M. Mourão Jr., $9 \AA$, $3 q$, $2 ð$ immat., 16 immat., 7 ov., (MPEG 12); idem, 13. vii.1995, idem, 19ð, $10 \bigcirc$, (MPEG 15); campus da UFPA, Igarapé Sapucajuba (POEMA), Belém, 22.xi.1994, idem, $9 \hat{\jmath}, 3$ + 5 immat., 7 immat., 1 ov., (MPEG 66); campus da UFPA, Igarapé Sapucajuba (POEMA), Belém, 19.vi.1995, R.A.F. Maia and M. Mourão Jr., $6 \hat{\jmath}, 3$, (MPEG 323); bica da APEG, Belém, 17.ix.1997, M.P. de Barros, F.R. Pimentel, S.S.B. da Silva and R.N. Bittencourt, 15 ${ }^{\lambda}, 119,19$ ov., (MPEG 455); Ilha de Marajó, Limoeiro do Ajuru (01ํ5ㄴ $44^{\prime \prime}$ S 49²2'51" W), 18.x.1997, B. Mascarenhas, D.G. Guimarães

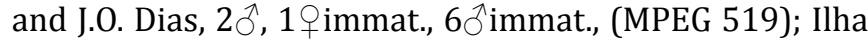
de Marajó, Muaná $\left(01^{\circ} 31^{\prime} 43^{\prime \prime}\right.$ S 49¹3'01" W), Furo do Palheta, 08.xi.1997, B. Mascarenhas and J.0. Dias, $2 \hat{\jmath}$, (MPEG 525); Rio Guajará, Ilha de São Domingos, São

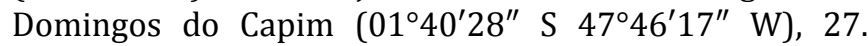
viii.1996, K. Nolan, 2ð, 1 + parasitada, (MPEG 137); Rio Caeté, community of Caeté, São Sebastião da Boa Vista

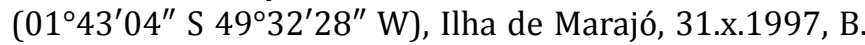
Mascarenhas, 1ㅇ, 1 ov., (MPEG 523); Rio Buiaçu, Ilha de Marajó, Breves (0140'57" S 50²8'50" W), 11.vi.1997, R.B. Neto, 3ð., (MPEG 256); Rio Pará, Ilha das Araras, Curralinho, 25.vii.1984, R. Barthem, $8 \hat{\jmath}, 1$, 3 qov., (INPA 1062); Rio Pará, Ilha das Araras, Curralinho, 24.vii.1984, R.

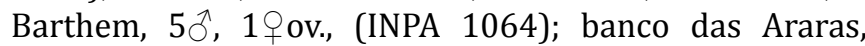
Curralinho, 24.vii.1984, R. Barthem, 3 ov., (INPA 1239); Rio Ipiranga, community of Ilha da Salvação, Melgaço

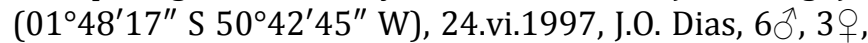
(MPEG 261); Rio Arrependido, tributary to Rio Caruta, community of Albuquerque, Melgaço $\left(01^{\circ} 48^{\prime} 17^{\prime \prime} \mathrm{S}\right.$ $50^{\circ} 42^{\prime} 45^{\prime \prime} \mathrm{W}$ ), 24.vi.1997, M. Zanuto, $1 \mathrm{O}^{\prime}, 1$ \% , (MPEG 258); ECFPn, FLONA de Caxiuanã, baía de Caxiuanã, Melgaço, 05.xi.1999, coll. unknown, 5今, 2ᄋ, (MPEG 727); idem, 03.xi.1999, idem, $3{ }^{\wedge}$ immat., 2 immat., 16juv., (MPEG

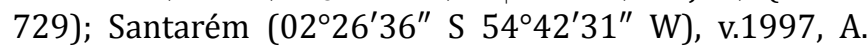
Magalhães, $1 \hat{\jmath}, 1$, (MPEG 208); Rio Tapajós, Santarém

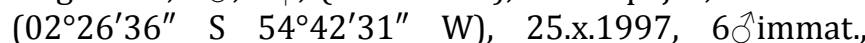
3 immat., (MPEG 518); Rio Tapajós, Santarém (02²6'36"

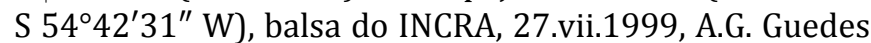
and A.L. Colares, $4 \hat{\bigcirc}, 3+, 5+$ ov., (MPEG 657); Rio Tapajós, mouth of Rio Maicá, Santarém ( $02^{\circ} 26^{\prime} 36^{\prime \prime}$ S 54 $42^{\circ} 31^{\prime \prime}$ W), 23.iv.1999, A.E.C. Gabelha, $1 \delta^{\lambda}, 2 \bigcirc, 2$ ov., (MPEG 659); community of Guajará, Santarém, 15.v.1999, coll. unknown, $2 \widehat{\jmath}, 4$ ㅇ, 1juv., (MPEG 665); Rio Tapajós, balsa do INCRA,

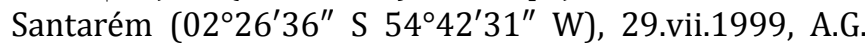
Guedes and A.L. Colares, $2 \hat{\bigcirc}, 1+1$, 1 ov., (MPEG 668); Rio 
Tapajós, Santarém (02²6'36" S 54²42'31" W), 31.vii.1999, R.N. Yuki, 1 ov., (MPEG 669); Comunidade de Santa Maria do Tapará, Santarém $\left(02^{\circ} 26^{\prime} 36^{\prime \prime} \mathrm{S} 54^{\circ} 42^{\prime} 31^{\prime \prime}\right.$ W), 21. xi.1999, coll. unknown, 10, 8 , (MPEG 679); community of São Jorge do Uricurituba, Santarém, 29.xii.1999, M.R.

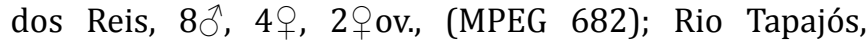

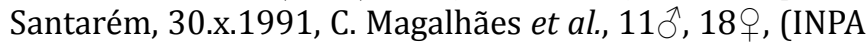
1197); Rio Tapajós, near Alter-do-Chão, 25.xi.1983, M. Goulding, 1 îmmat., 5 qimmat., (INPA 093); Rio Tapajós, near mouth of Rio cupari, 27.x.1991, A. Negrão et al., 2ठ, 13 immat., 30 Oimmat., (INPA 1175); Rio Tapajós, unnamed igarapé on the right bank near mouth of Rio Cupari-São Luis, 26.x.1991, C. Magalhães and J. Zuanon, 5 Pimmat., (INPA 1187); Rio Tapajós, Alter-do-Chão, 28.x.1991, J. Zuanon, E.N. Santos Silva and C. Magalhães, 1ð, 1․ (INPA 1190); Rio Trombetas, Lago Abui, 11. iii.1986, V. Py-Daniel et al., $2 ð$ immat., 10 immat., (INPA 401); Lago Jacundá, Alter-do-Chão, Santarém, 16.ii.2000, C.R. García-Dávila, $1 \widehat{\delta}$, (INPA 1105); headwaters of Lago Verde, Alter-do-Chão, Santarém, 18.ii.2000, C.R. GarcíaDávila, 23 immat., (INPA 1240); headwaters of Lago Verde, Alter-do-Chão, Santarém, 18.ii.2000, C.R. GarcíaDávila, 1§, (INPA 1131); Lago Jucuruí, Alter-do-Chão, Santarém, 21.ii.2000, C.R. García-Dávila, 7 đ̂, 6q, 4 qimmat., (INPA 1141); Lago Piranha, Alter-do-Chão, Santarém, 19. ii.2000, C.R. García-Dávila, $27 \bigcirc, 18$, $7 \bigcirc$ immat., 8 @immat., (INPA 1138); Rio Tocantins, Tucuruí, 28.i.1985, 0. OdinetzCollart, 20今, $2 \circ, 9+$ ov., (INPA 202); idem, 17.iv.1988, U. Barbosa, $2{ }^{\Uparrow}$ immat., 4 immat., (INPA 1221); idem, Tucuruí Dam, 23.vii.1987, idem, $3 \widehat{\text { }}$ immat., (INPA 1222); idem, Igarapé Cametá, 12.vii.1987, idem, 19immat., (INPA 1223); BR-422 road, Rio Ararão, 22.iv.1988, idem, 2 immat., 3 immat., (INPA 1225); idem, Acaripucu, 13. vii.1987, idem, $1 \delta^{\text {immat., } 2} 2$, 7 o ov., (INPA 1226); idem, 18.vii.1987, idem, $2{ }^{\top}$ immat., 6 immat., 1 im., 4 juv., (INPA 1228); idem, 30.iv.1987, idem, $3 \hat{\jmath}, 1$ P immat., (INPA 1229); idem, 16.vii.1987, idem, $2 \widehat{\jmath}$ immat., 34 ९ immat., 1 ov., (INPA 1233); idem, left bank, 12.vii.1987, idem, 5 کैimmat., 1 immat., (INPA 1234); idem, Icangui, 01.xii.1987, idem, 5 immat., 5 @immat., 4juv., (INPA 1231); idem, idem,

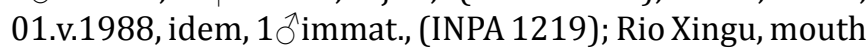
of Maxacá, 20.x.1992, M. Jegu, 5 îmmat., 3 i immat., (INPA 1156); idem, Paraná Aramaná, 16.x.1992, 0. OdinetzCollart, 11 îmmat., 9 immat., 1 iov., (INPA 1168); Ilha do Cipó, lower Rio Xingu, Pitanga, 26.xi.1983, P. Sá and B. Mascarenhas, $7 \hat{\sigma}, 5$, (MPEG 75). All records are shown in Figure 4 .

\section{Macrobrachium brasiliense (Heller, 1862)}

Palaemon brasiliensis Heller 1862: 419, pl. 2, fig. 46.

Macrobrachium brasiliense - Coelho and Ramos-Porto 1985: 408 [in list]; Barros and Pimentel 2001: 20 [in list]; Melo 2003: 346 [map]; Magalhães and Pereira 2007: 14 [in list]; Vieira 2008: 69 [in list].

Geographic distribution. Venezuela, Colômbia, Guyana, Suriname, French Guiana, Ecuador, Peru, Brazil (Acre, Amapá, Pará, Amazonas, Maranhão, Bahia, Mato Grosso, Mato Grosso do Sul, Goiás, Bahia, Minas Gerais, São Paulo, Paraná) (see Pileggi et al. 2013 for references).

Material examined. AMAPÁ: Rio Davi Grande, lef tributary of Rio Água, Amapá, 14.iv.1997, I. Assunção, 1

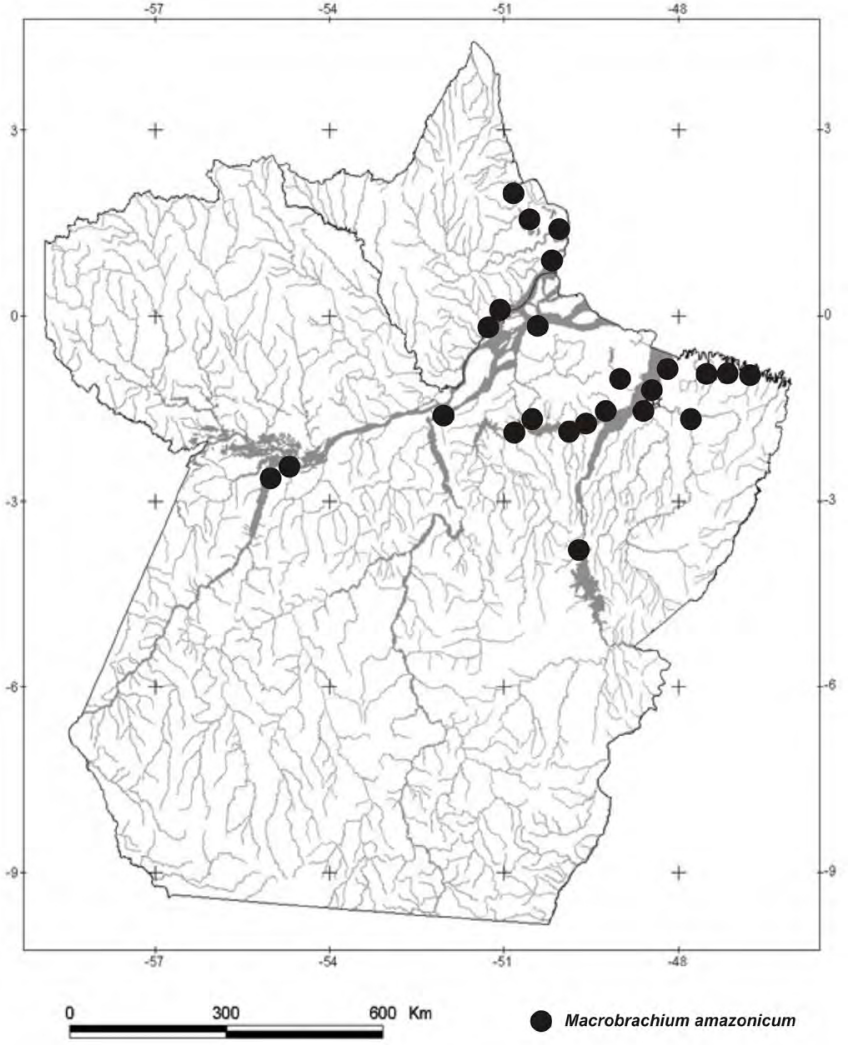

FigurE 4. Geographic distribution of Macrobrachium amazonicum (Heller, 1862) in the States of Amapá and Pará, Brazil (some symbols may represent more than one record).

spec., (MPEG 230); Rio Tracajatuba crossing the road BR 156, Amapá, 15.iv.1997, I. Assunção, 1ठ̄. (MPEG 232); BR-156 road, Furo do Henrique, Amapá, 27.viii.1992, C. Magalhães, 2ð, 1\%, $7 \lesssim$ immat., 16 @immat., 9juv., (INPA 1072); Rio Amapá Grande, Cachoeira Grande, Amapá, 26.viii.1992, C. Magalhães, 3ㅇ, (INPA 1076); Rio Araguari, igarapé near Cachoeira da Capivara, Amapá, 20.viii.1992, C. Magalhães et al., 10 đ, 6 + immat., $5 \bigcirc$ immat., 15juv., (INPA 1074); Rio Araguari, Marcírio, upriver from Porto Grande, 18.viii.1992, C. Magalhães and M. Jegu, 1․ (INPA 1070); Serra do Navio, Igarapé Água Branca, 22.iii.1991, V. Py-Daniel et al., 2 †immat., (INPA 1211); BR-210, Igarapé Munguba, Macapá, 23.iii.1991, V. PyDaniel et al., 3 Pimmat., (INPA 1207); BR-210, Igarapé Água Fria, Macapá, 22.iii.1991, V. Py-Daniel et al., 1ठ, 1 immat., (INPA 1209); road to Projeto Cromita, Rio Vila Nova, 23.iii.1991, V. Py-Daniel et al., 2 +immat., (INPA 1210); road Macapá-Jari, Igarapé Cachoeira, Amapá,

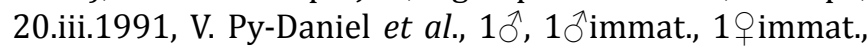
(INPA 1213); Igarapé do Marinho, Laranjal do Jari,

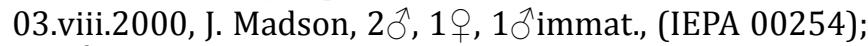
PARÁ: praia de Ajuruteua $\left(00^{\circ} 51^{\prime} 337^{\prime \prime} \mathrm{S} 46^{\circ} 36^{\prime} 050^{\prime \prime} \mathrm{W}\right)$, iii.1986, B. Mascarenhas, $3 \widehat{\jmath}$. (MPEG 115); Inajá, Igarapé Vilemar, Primavera, 07.ix.1987, B. Mascarenhas et al., 1 , 4 îmmat., 6 Pimmat., (MPEG 757); junction of Igarapé Tramalhetinho with Rio Trombetas, 02.xi.1985, L.M. dos

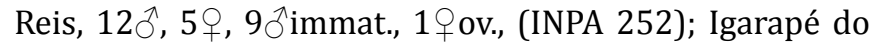
Km 10, BR-163 road, near Cachoeira Porteira, 07.x.1985, C. Magalhães, $1 \hat{\jmath}, 6 \hat{\jmath}$ immat., 7 immat., $1 q$ ov., (INPA 334); Rio Trombetas, igarapé at Estirão da Fumaça, 14.x.1985, C. Magalhães, $2 \hat{\jmath}, 17 \delta$ immat., 20 immat., $2+$ ov., (INPA 339); Rio Trombetas basin, igarapé tributary to Rio Coxi-

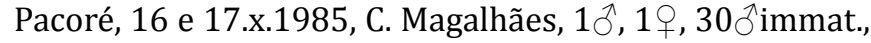


37 immat., 4juv., (INPA 341); Igarapé do Cipó, Castanhal,

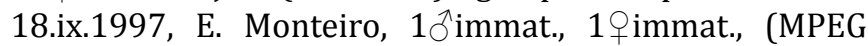
452); Colônia do Cupiúba, Igarapé do Cipó, Castanhal, 14.vi.1999, D. Guimarães, 19, (MPEG 700); road to Genipaúba, Morelândia Farm, Benevides (0121'42" S 48¹4'42" W), 19.ix.1998, A. Quaresma and D. Guimarães, $1 \hat{\jmath}, 1$ immat., (MPEG 583); road PA-140, Km 465, leaving Bujaru, 25.ix.1997, E. Monteiro, 1 immat., (MPEG 465);

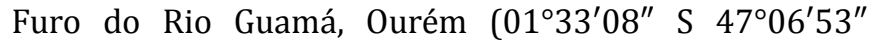

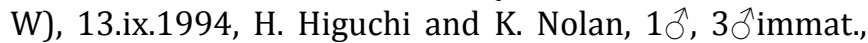
4 immat., (MPEG 187); Igarapé Poraquequara, Ourém

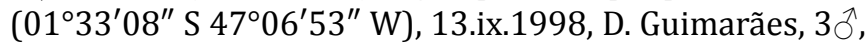
(MPEG 584); rodovia Trans-Cametá, unnamed igarapé, 08.vi.1988, U. Barbosa, 1ठ̂, (INPA 1224); BR-230 road, 23.iv.1988, U. Barbosa, 1 immat., (INPA 1227); Fazenda Cauaxi, Paragominas, 15.iv.1999, R.M. dos Santos, 1q, (MPEG 699); Rio Xingu, Altamira, 15.xii.2000, R. Souza and Osmar, $1 \hat{\jmath}, 10 \AA$ immat., 4 ○ immat., (MPEG 709); idem, 16.xii.2000, R. Souza and Masinho, 2 immat., (MPEG 710); idem, idem, 17.xii.1999, R. Souza and Cristiana, 4§. (MPEG 712); idem, Ilha do Forno, idem, 18.xii.2000, R. Souza and

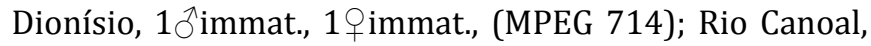
right bank tributary to Rio Tocantins, $35 \mathrm{Km} \mathrm{S}$ of Tucuruí (0345'59" S 4940'22" W), 28.iii.1984, B. Mascarenhas and A.L. Nunes, 10․ (MPEG 91); Rio Tocantins, Tucuruí Dam, 23.vi.1984, W.L. Overal, 1 immat., (MPEG 95); Rio Tocantins, Igarapé Arapari, Breu Branco, 13.vii.1982, coll. unknown, 10َ, (INPA 277); Rio Xingu, Igarapé do Ipixuna,

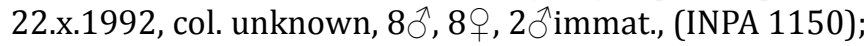
Rio Tocantins, Itupiranga, 09.xi.1985, 0. Odinetz-Collart, $1 \hat{\jmath}, 1$ immat., (INPA 384); Rio Xingu, Igarapé Triunfo, 20.x.1992, col. unknown, 15 îmmat., 40 年immat., (INPA 1157); idem, Cachoeira Gorgulho do Espelho, 12.x.1992,

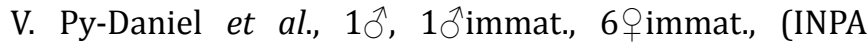
1152); idem, Cachoeira do Quataquara, 15.x.1992, coll. unknown, 10̄, (INPA 1153); Rio Pardo, 21.x.1992, coll. unknown, $1 \hat{\jmath}, 2 \widehat{\jmath}$ immat., 24 immat., (INPA 1154); idem, Ilha de Babaquara, 05.x.1990, J. Zuanon, 16ㅇ, $8{ }^{\top}$ immat., 49 immat., (INPA 1162); idem, 30.ix.1990, coll. unknown, 1, 2 immat., 1 immat., (INPA 1163); idem, 30.ix.1990, idem, $11 \hat{\jmath}, 14 \delta$ immat., 16 †immat., (INPA 1164); idem, Igarapé do Bom Jardim, 21.x.1992, coll. unknown, 1ð̂, 6 Pimmat., (INPA 1166); idem, Lago do Poção, Igarapé do Poção, 11.x.1992, V. Py-Daniel et al., 2 $\widehat{O}$, (INPA 1170); idem, Lago do São Sebastião, 18.x.1992, coll. unknown,

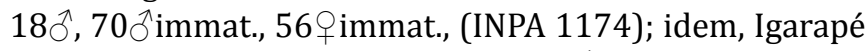
do Panta, 21.x.1992, coll. unknown, 1§̂, 2 @immat., (INPA 1217); idem, abaixo de Buburé, 22.x.1991, J. Zuanon et al., 2 ○̂immat., 2 ○immat., 4juv., (INPA 1181); idem, just downstream from Buburé, 22.x.1991, C. Magalhães, 2今, 2 , (INPA 1182); Rio Tapajós, downstream from Buburé, 22.x.1991, C. Magalhães et al., 6ภ, 5 îmmat., 3 immat., 5juv., (INPA 1195); community of Santo Antônio, Itaituba,

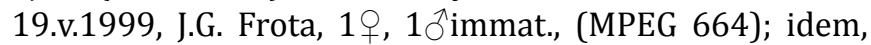
11.x.1999, idem, 3 $\hat{\jmath}$, (MPEG 676); Rio Jamanxim, unnamed igarapé, 20.x.1991, C. Magalhães, 11 $\delta^{1}, 46+$ immat., $1 q$ ov., (INPA 1179); idem, Ilha da Terra Preta, 20.x.1991, C.

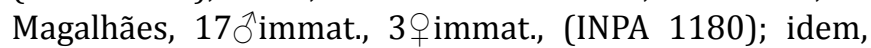
Ilha da Terra Preta, 20.x.1991, Raimundinho, $4{ }^{\lambda}$ immat., 12 immat., 2juv., (INPA 1184); idem, channel near Ilha da Terra Preta, 20.x.1991, C. Magalhães et al., 7 immat., (INPA 1193); idem, Ilha da Terra Preta, 21.x.1991, C.
Magalhães, $6 \hat{\jmath}, 7$ immat., 5 immat., (INPA 1196); Serra das Andorinhas, Cachoeira do Spaner, São Geraldo do Araguaia, 12.v.2001, I.S. Gorayeb, $4 \hat{\jmath}, 1$ immat., (MPEG 753); Rio Parauapebas, Mineração Serra do Sossego, Carajás, 25.iv.2002, R.P. Ribeiro, 1 $\hat{\jmath}, 8$, $4 \hat{\jmath}$ immat., 7 immat., 2 ov., 1juv., (INPA 1236); idem, Mineração Serra do Sossego, Carajás, 12.vi.2002, R.P. Ribeiro, 7ð, 1 ㅇ, 1 îimmat., 3 immat., 2 ov., (INPA 1237); Rio Salobo, Serra Norte de Carajás, 27.iv.1983, col. unknown, 2 exuviae, (MPEG 97); Balneário Atay, Monte Dourado, 09.iv.2002, B. Mascarenhas et al., 1 immat., (MPEG 746). All records are shown in Figure 5.

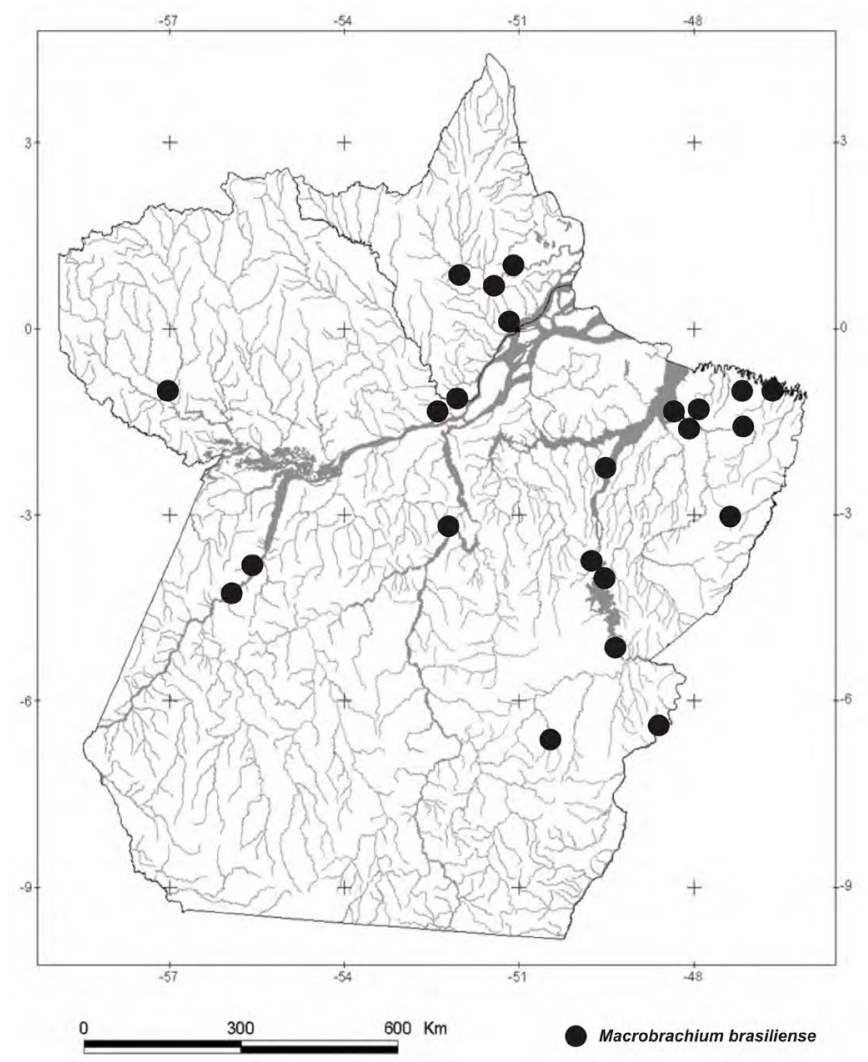

FiguRE 5. Geographic distribution of Macrobrachium brasiliense (Heller, 1862) in the States of Amapá and Pará, Brazil (some symbols may represent more than one record).

Macrobrachium carcinus (Linnaeus, 1758)

Cancer Carcinus Linnaeus 1758: 631.

Macrobrachium carcinus - Ramos-Porto and Coelho 1998: 333 [in list]; Barros and Pimentel 2001: 20 [in list]; Melo 2003: 348 [map]; Pileggi and Mantelatto 2010: 196 [in list]; Cavalcante et al. 2012 [in list].

Geographic distribution. Antilles, North America, Central America and South America, from Venezuela to Brazil (from Pará to Rio Grande do Sul) (Holthuis 1952; Delgado et al. 1997; Coelho and Ramos-Porto 1998; Bowles et al. 2000).

Material examined. AMAPÁ: Arquipélago do Bailique, mouth of Igarapé do Faustino, Amapá, 31.viii.1995, L. Ramos, J. Cardoso and N. Farripas, $1 \hat{\jmath}$, (IEPA 00048); Arquipélago do Bailique, Amapá, 18.vi.2000, I.M. Vieira,

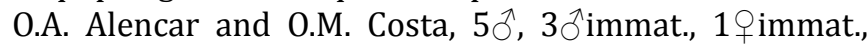
(IEPA 00221); Fazendinha, between SENAVA shipyard and Cascalheira, Macapá, 26.v.1990, J.C.S. Oliveira, 2へ̂. (IEPA 00010); Rio Amazonas (left bank), between Fazendinha 
and Cascalheira, Macapá, 06.v.1990, J.C.S. Oliveira, 107 , 1 ov., (IEPA 00017); Ilha de Santana, Santana, 15.iv.2000, I.M. Vieira, O.A. Alencar, O.M. Costa, J. Cardoso and A.C. de Souza, $1 \delta^{\text {immat., }} 3$ immat., (IEPA 00208); Igarapé da Fortaleza, Santana, 04.ii.1997, 0.M. Costa, 1 đ̂, 1 đimmat., (IEPA 00125). PARÁ: Ilha do Pará, Igarapé do Anta, Afuá, 11.xii.2000, I.M. Vieira, O.M. Costa, O.A. Alencar and D.A. Halboth, 1 spec., (IEPA 00235); Rio Pracuúba, Ilha do Pará, Afuá, 18.xi.1998, I.M. Vieira, O.A. Alencar and O.M. Costa, 1 đิ. (IEPA 00258); Rio Maniva, Ilha do Pará, Afuá, iii.1999, coll. unknown, 1 spec., (IEPA 00183); idem, iv.1999, 0.M. Costa, O.A. Alencar and J. Cunha, 1 spec., (IEPA 00184); Praia de Colares, Colares, 28.iii.1997, A. Trindade, 10 , (MPEG 173); Rio Gurupi, Viseu, xi.1993, A.C.M. Lima and R.A.T. Rocha, $1 \delta^{\lambda}$ (exúvia), (MPEG 341); Rio Tocantins, Cametá, 15.iii.1985, 0. Odinetz-Collart, 1ð,1 1 , (INPA 283); idem, 15.i.1985, idem, 1ठ̄. (INPA 262); Joroca, Cametá (02¹4'41" S 49²9'46" W), 10.xi.1996, J.R. Carvalho Jr.,

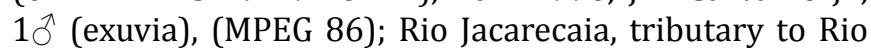
Tocantins, Mocajuba, 23.x.1952, 0. Rego, 1, (MPEG 87). All records are shown in Figure 3.

\section{Macrobrachium jelskii (Miers, 1877)}

Palaemon jelskii Miers 1877: 661, pl. 67, fig. 1.

Macrobrachium jelskii - Coelho and Ramos-Porto 1985: 408 [in list]; Ramos-Porto and Coelho 1998: 334 [in list]; Barros and Pimentel 2001: 20 [in list]; Vieira 2003: 61, fig.; Melo 2003: 362 [map].

Geographic distribution. Trinidad, Venezuela, Suriname, French Guiana, Peru, Bolivia, Argentina, Brazil (Amapá, Amazonas, Pará, Maranhão, Ceará, Rio Grande do Norte, Paraíba, Pernambuco, Alagoas, Sergipe, Bahia, Acre, Mato Grosso, Espírito Santo, Rio de Janeiro, Minas Gerais, São Paulo, Paraná) (see Pileggi et al. 2013 for references).

Material examined. AMAPÁ: Lago Pracuúba, Amapá, vii.1984, M.A.S, Lima, $5 \bigcirc \hat{\jmath}, 14$ @ , 13 ov., 1juv., (IEPA 00006); headwaters of Rio Sucuriju, Amapá, xi.1996, Raimundinho, O.M. Costa and I.M. Vieira, 1, 2juv., (IEPA 00130); idem, xi.1997, I.M. Vieira, O.M. Costa and Raimundinho, 66 $36 \circ$, 99 ov., 15juv., (IEPA 00131); district of Sucuriju, Lago Piratuba, Amapá, ix.1997, I.M. Vieira, 1 spec., (IEPA 00141); idem, x.1997, J. Cardoso, I.M. Vieira and O.M. Costa, 1110̂, 59 , (IEPA 00142); idem, 21.iii.1998, I.M.

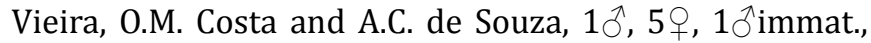
69 ov., (IEPA 00164); headwaters of Rio Sucuriju, district of Sucuriju, Amapá, xi.1997, I.M. Vieira, O.M. Costa and J. Cardoso, $2 \hat{\jmath}, 1$ immat., (IEPA 00175); headwaters of Rio Sucuriju, district of Sucuriju, Amapá, xi.1997, I.M. Vieira and Raimundinho, 3 immat., 8 immat., (IEPA 00197); headwaters of Rio Sucuriju, Amapá, xi.1997, I.M. Vieira and Raimundinho, 2 immat., (IEPA 00245); Rio Araguari, Lago Piratuba Biological Reserve, channel to the lakes, 16.viii.1992, C. Magalhães, $37 \hat{\jmath}, 3+, 1 \delta$ immat., 5juv., (INPA 1065); Lago Pracuúba, Amapá, vii.1984, M.A.S. Lima, 1 , 1 ov., (INPA 1066); Rio Amapá Grande, Cachoeira Grande, Amapá, 25.viii.1992, C. Magalhães, 10 đ̃, 2 qimmat., 2juv., (INPA 1060); 1ㅇ,1ㅇov., (MPEG 231); Ressaca do Tacacá, Macapá, 29.vi.2000, N. Crud and C. Robds, 4ð̄, (IEPA 00253); Macapá, ii.1984, M. Goulding, $3{ }^{7}, 8$,, 4 ovov., (INPA 207). PARÁ: Santarém-Novo ( $\left.00^{\circ} 55^{\prime} 45^{\prime \prime} \mathrm{S} 47^{\circ} 23^{\prime} 50^{\prime \prime} \mathrm{W}\right)$,
Igarapé Bacuri, 26.iv.1998, F.R. Pimentel and R.N. Yuki, $2 \bigcirc, 2 \bigcirc$ ov., (MPEG 564); Inajá, Igarapé Vilemar, Primavera, 07.ix.1987, B. Mascarenhas et al., 1, $5 \bigcirc$ immat., 6 immat., 3 ov., (MPEG 758); idem, 23.v.1987, B. Mascarenhas

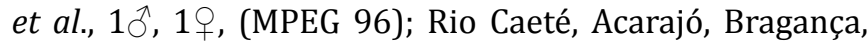
28.vi.1996, A. Montenegro and V.J. Isaac, 3 spec., (MPEG 180); Sítio Aracuã, Jequiri, between Bragança and AugustoCorrêa, 15.xi.1999, F.R. Pimentel and R. Souza, 18 $\sigma^{2}, 7$, 12 ov., (MPEG 786); idem, ii.1999, L.M.A. da Silva, 1spec.,

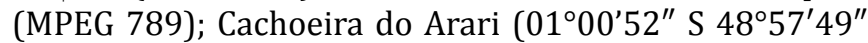
W), mouth of Rio Goiapi, Ilha de Marajó, 28.x.1990, I. Assunção, 10َ, (MPEG 83); idem, 08.iv.1990, idem, $13 \hat{\jmath}$, 4 ㅇ, 4 qov., (MPEG 84); idem, 11.iv.1990, idem, 54へ, 38 ,

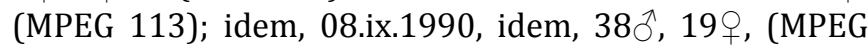
136); Rio Piracatuba near Ilha do Mosqueiro, 29.x.1984, J. Porto and P. Rocha, 2 $\hat{0}, 3$ immat., (MPEG 103); bica da APEG, Belém, 17.ix.1997, M.P. de Barros, F.R. Pimentel, S.S.B. da Silva and R.N.Bittencourt, $27 \partial^{\lambda}, 19$, (MPEG 456); Rio Guamá, lagoa velha, Ourém $\left(01^{\circ} 33^{\prime} 08^{\prime \prime}\right.$ S 47 $06^{\prime} 53^{\prime \prime}$ W), 29.viii.1993, J. Carvalho Jr., $10^{\wedge}$, (MPEG 102); Furo do

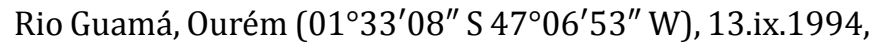
H. Higuchi and K. Nolan, 11 , (MPEG 185); idem, 1 , (MPEG 333); Rio Amazonas, São Raimundo, Almeirim, 12.v.1999, J. Carvalho Jr., $1 \delta$, 1 q , 1 q ov., (MPEG 686); idem, Pesqueiro, quadra 32, idem, 26.vii.1999, idem, 28 9 , 9 , 5 ov., (MPEG 688); Rio Araiollos, Rio Amazonas Basin, Pesqueiro São Paulo, Almeirim, 27.vii.1999, idem, 10, 4, (MPEG 690); Rio Espusento, Almeirim, 28.vii.1999, idem, 19, (MPEG 692); ECFPn, FLONA Caxiuanã, Rio Arauá, Melgaço, 25.vi.1997, J.A.R. Bernardi and R.A.J. Rocha, 1ㅇ, (MPEG 305); idem, 22.vii.1997, C. Roque and D.C. Pimentel Neto, $3 \hat{\jmath}, 6 \circ, 2$ immat., 4 ov., (MPEG 383); idem, Rio Curuá, Melgaço, 29.i.1998, J.A.R. Bernardi, 49 ov., (MPEG 576); idem, mouth of Rio Caxiuanã, Melgaço, 09.xi.1999, coll. unknown, $4 \hat{\jmath}, 10 \uparrow, 4$ ov., (MPEG 730); idem, mouth of Rio Curuá, Melgaço, xi.1999, idem, 18 ${ }^{\hat{N}}$, 20 +, 5 immat., 1 o ov., (MPEG 731); idem, 02.vii.2000, Ferreira and Adélia, 3 spec., (MPEG 755); idem, Melgaço, vii.2000, B. Mascarenhas, 1 , $5 \bigcirc$ immat., $7 \uparrow$ immat., $1 q$ ov., 1juv., (MPEG 761); idem, Rio Curuá, Melgaço, 09.vii.2000, B. Mascarenhas, $1 \hat{\jmath}, 10,9$ immat., (MPEG 762); idem, Rio Curuá, Melgaço, 07.vii.2000, B. Mascarenhas, 10̄, 6 immat., (MPEG 763); idem, Enseada São Raimundo, Rio Curuá, Melgaço, 01.x.2000, Adélia, 2ð, 4 †immat., (MPEG 768); idem, Porto da Estação, Melgaço, 22.iv.2000, coll. unknown, 14 immat., (MPEG 769); Ilha do Combú, Acará, 24.x.2001, Adélia, 14 spec., (MPEG 771); Laguinho, Óbidos, 12.viii.1984, I. Assunção, 4ㅇ, (MPEG 106); Rio Curuá do Sul, near Santarém, 15.xi.1977, coll. unknown, 9ð, 11, (INPA 117); Rio Xingu, mouth of Maxacá, 20.x.1992, M. Jegu, $1 \oint^{\lambda}, 4$, $11 \overbrace{}^{\lambda}$ immat., 10 qimmat., (INPA 1155); idem,

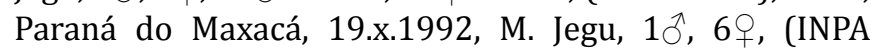
1158). All records are shown in Figure 6.

\section{Macrobrachium nattereri (Heller, 1862)}

Palaemon Nattereri Heller 1862: 414, est. 2, fig. 36.

Macrobrachium nattereri - Holthuis 1952: 83, pl. 20, figs. a-d; Holthuis 1966: 3; Kensley and Walker 1982: 9, figs. 11, 12d [Tucurí = ?Tucuruí]; Ramos-Porto and Coelho 1998: 334 [in list]; Barros and Pimentel 2001: 20 [in list]; Melo 2003: 324 [map]; Magalhães and Pereira 2007: 14 [in list]. 
Geographic distribution. Venezuela, Colombia, French Guiana, Brazil (Amazonas, Pará, Ceará, Bahia, Goiás, Mato Grosso) (see Pileggi et al. 2013 for references).

Material examined. PARÁ: mouth of Rio Caeté, Bragança, 22.viii.1995, A. Montenegro and V.J. Isaac, 5 îmmat., 1 immat., (MPEG 183); Cachoeira Porteira, Igarapé São Miguel, 04-06.x.1985, C. Magalhães, 63 , 20 îmmat., 3 immat., (INPA 335); Rio Trombetas basin, Igarapé Patauá, tributary to Rio Madeira, 09-21.x.1985, C.

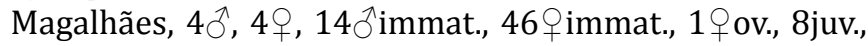
(INPA 342); Vila do Conde, Linhão, Barcarena, 25.iii.2002, B. Mascarenhas et al., 2 @immat., (MPEG 750); Balneário

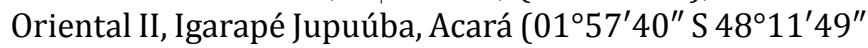
W), 08.x.1997, G.J.A. Silva, 2ð, 5 †immat., 10juv., (MPEG 515); Ilha do Combú, Acará, 24.x.2001, Adélia, 123 immat., 11 immat., (MPEG 770); Lago Jacundá, Alter-do-Chão, Santarém, 16.ii.2000, C.R. García-Dávila, 2+, 10 †immat., (INPA 1104); Igarapé do Irurama, idem, 17.ii.2000, C.R.

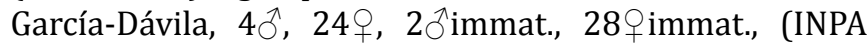
1117); Igarapé São Sebastião, idem, 17.ii.2000, C.R. García-Dávila, $15 \hat{\jmath}, 9 q, 9 \widehat{\text { immat., }} 41$ immat., $1 q$ ov., (INPA 1120); headwaters of Lago Preto, idem, 17.ii.2000,

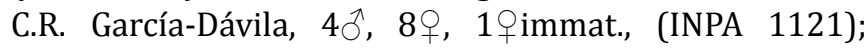
headwaters of Lago Verde, idem, 18.ii.2000, C.R. GarcíaDávila, $2 \hat{\jmath}, 2$, (INPA 1128); Lago Verde, idem, 18.ii.2000, C.R. García-Dávila, 1ð̄, (INPA 1134); Rio Tapajós, near mouth of Rio Cupari, 26.x.1991, C. Magalhães, J. Zuanon and E.N. Santos Silva, 2へ, 4 ○immat., (INPA 1185); idem, Itaituba, 18.x.1991, C. Magalhães et al., 19, (INPA 1188); idem, mouth of Rio Maicá, Santarém $\left(02^{\circ} 26^{\prime} 36^{\prime \prime} \mathrm{S}\right.$

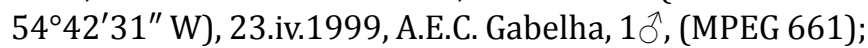
Rio Tocantins, small rapids downstream from Jatobal and Timbó, 08.vii.1982, coll. unknown, $2 \jmath^{3}$ immat., 1 immat., (INPA 1067); idem, Tucuruí Dam (0345'59" S 4940'22" W), 24.vi.1984, W.L. Overal, 1 immat., (MPEG 107); idem, 27.vi.1984, idem, $1 \widehat{\jmath}$, (MPEG 108); Serra das Andorinhas, São Geraldo do Araguaia, 23.vii.2000, B. Mascarenhas et al., 4 qimmat., (MPEG 737); Igarapé do Fofoca, Serra dos Carajás, 18.iv.1983, B. Mascarenhas, 2ðimmat., (MPEG

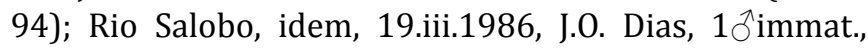
(MPEG 98); Igarapé Pojuca, idem, 21.vi.1985, M. Zanuto, 1 immat., (MPEG 99); Serra Norte dos Carajás, 21.iii.1985, R.N. Bittencourt, $1 \delta^{\wedge}$ immat., (MPEG 109); Rio Itacaiuna, Km 07 of road to Caldeirão, idem, 23.iv.1983, coll. unknown, 2 immat., 6 immat., (MPEG 110); Rio Itacaiuna, Km 13 of road to Caldeirão, idem, 23.iv.1983, coll. unknown, 1 , (MPEG 111); Rio Jamanxin, unnamed igarapé, 20.x.1991, C. Magalhães, $1 \hat{\jmath}, 1$, (INPA 1178); idem, Ilha da Terra Preta,

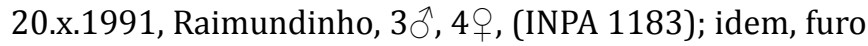
near Ilha da Terra Preta, 20.x.1991, C. Magalhães et al., $17 \hat{\jmath}, 49+$, (INPA 1192). All records are shown in Figure 6.

\section{Macrobrachium olfersii (Wiegmann, 1836)}

Palaemon Olfersii Wiegmann 1836: 150.

Macrobrachium olfersi - Vieira 2008: 69 [in list]; Melo 2003: 366 [map].

Geographic distribution. USA (Florida, Mississipi, Texas), islands of Caribbean, Gulf of México, Guatemala, Venezuela, Brazil (from Piauí to Rio Grande do Sul) (Holthuis 1952; Delgado et al. 1997; Coelho and RamosPorto 1998; Bowles et al. 2000).

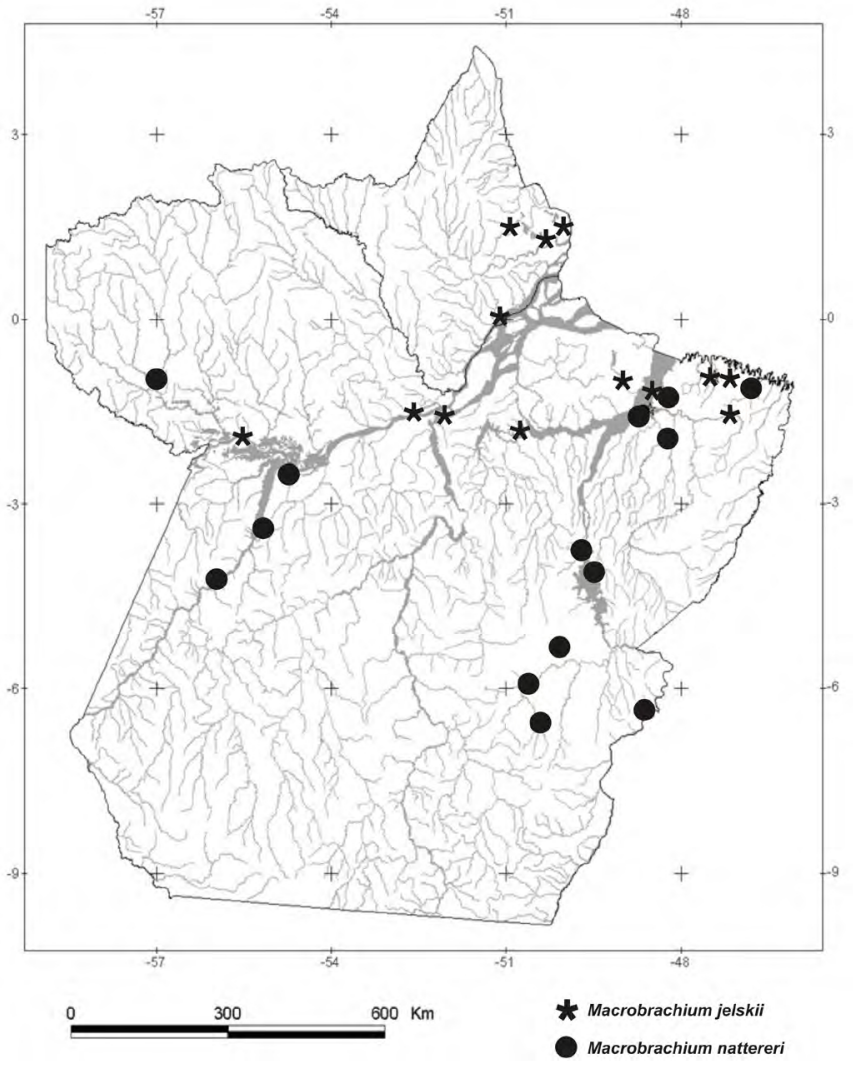

FIGURE 6. Geographic distribution of Macrobrachium jelskii (Miers, 1877), and M. nattereri (Heller, 1862) in the States of Amapá and Pará, Brazil (some symbols may represent more than one record).

Material examined. AMAPÁ: headwaters of Rio Sucuriju, Amapá, xi.1997, I.M. Vieira and Raimundinho, 13오 (IEPA 00173); idem, xi.1996, Raimundinho, O.M. Costa and I.M. Vieira, 2 ov., (IEPA 00244); idem, xi.1997, Raimundinho, O.M. Costa and I.M. Vieira, 1 ov., (IEPA 00246); Vila de Sucuriju, Amapá, 18.iv.2001, I.M. Vieira and O.M. Costa, 2 ov., (IEPA 00262); Rio Amapá Grande, Cachoeira Grande, Amapá, 26.viii.1992, C. Magalhães, 9 đimmat., 3 immat., 2 ov., 3juv., (INPA 1073); idem, 25.viii.1992, C. Magalhães, 1ð̂, 1 ov., (INPA 1075); idem, 25.viii.1992, C. Magalhães et al., 17juv., (INPA 1077); idem, 25.viii.1992, C. Magalhães et al., $2 \hat{\jmath}, 1$ immat., 44juv., (INPA 1078); idem, viii.1992, coll. unknown, 3 , , (INPA 1103). All records are shown in Figure 3.

\section{Macrobrachium surinamicum Holthuis, 1948}

Macrobrachium surinamicum Holthuis 1948: 1112; Coelho and Ramos-Porto 1985: 408 [in list]; Ramos-Porto and Coelho 1998: 335 [in list]; Barros and Pimentel 2001: 20 [in list]; Melo 2003: 372 [map]; Pileggi and Mantelatto 2010: 196 [in list]; Cavalcante et al. 2012 [in list].

Geographic distribution. Venezuela, Guyana, Suriname, Brazil (Amapá, Pará) (see Pileggi et al. 2013 for references). Material examined. AMAPÁ: Rio Amapá Grande, Cachoeira Grande, Amapá, 26.viii.1992, C. Magalhães, 19 ov., 1juv., (INPA 1071); Arquipélago do Bailique, Amapá, 21-26.vi.2000, I.M. Vieira, O.A. Alencar and O.M. Costa, $16 \overbrace{}^{\lambda}$, $5 \bigcirc, 2$ ov., (IEPA 00219); Cubana, Arquipélago do Bailique, Amapá, 23.ix.2000, I.M. Vieira, O.A. Alencar, O.M. Costa and

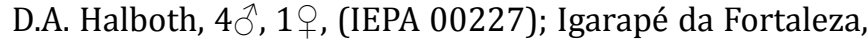


Santana, vi.1990, J.C.S. Oliveira, $1 \hat{\delta}$, (IEPA 00022); idem, vi.1990, coll. unknown, $2 \hat{\jmath}$, (IEPA 00023); Ilha de Santana, Santana, 15.iv.2000, I.M. Vieira, O.A. Alencar, O.M. Costa, J. Cardoso and A.C. de Souza, 1 ov., (IEPA 00250). PARÁ: Ilha Rosa-Chagas, municipality of Pará, 23.iii.1998, coll. unknown, 7ð, 11오. (IEPA 00170); Ilha Gurupá, Igarapé

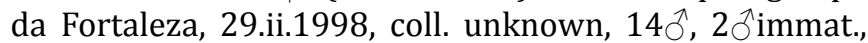
2 ov., (IEPA 00171); Rio Maniva, Ilha do Pará, Afuá, 26.iv02.v.1999, O.A. Alencar, O.M. Costa and J. Cunha, 42 $̋, 24$, 39 ov., (IEPA 00178); idem, 18-20.vi.1999, O.M. Costa, O.A.

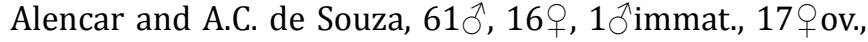
(IEPA 00185); idem, 16.xii.1999, I.M. Vieira, O.M. Costa and O.A. Alencar, $34 \circlearrowleft, 12 \circ, 4$ ov., (IEPA 00195); idem, mouth of Igarapé do Anta, Afuá, 13 e 14.iv.2000, I.M. Vieira, O.A. Alencar, J. Cardoso, O.M. Costa and A.C. de Souza, 15 $\overbrace{}^{\lambda}, 9$, 15 ov., (IEPA 00204); idem, 14 e 15.iv.2000, I.M. Vieira,

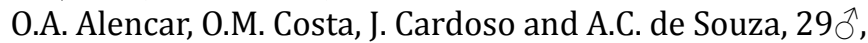
5 9 , 2 immat., 19 ov., (IEPA 00205); idem, 14-15.iv.2000, I.M. Vieira, O.A. Alencar, O.M. Costa, J. Cardoso and A.C. de Souza, 8$\hat{\jmath}, 16 q, 16 q$ ov., (IEPA 00206); idem, 16.iv.2000, I.M. Vieira, O.M. Costa, O.A. Alencar, J. Cardoso and A.C. de Souza, $17 \lesssim, 9 q, 35$ ov., (IEPA 00209); idem, 18.vi.2000, I.M. Vieira, O.A. Alencar and O.M. Costa, $43 \hat{\jmath}, 31$,, $18 q$ ov., (IEPA 00218); idem, 07-12.xii.2000,I.M.Vieira, O.A. Alencar, O.M. Costa and D.A. Halboth, 15 $\hat{\circ}$, (IEPA 00234); idem, 14.ii.2001, coll. unknown, $5 \hat{\jmath}, 2$ ov., (IEPA 00237); Rio Maniva, Ilha do Pará, Afuá, 03 e 04.xii.2000, I.M. Vieira, O.A.

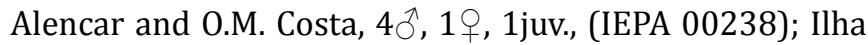
do Pará, Igarapé do Anta, Afuá, 19-25.vi.2001, I.M. Vieira, O.A. Alencar and O.M. Costa, $50 \hat{\jmath}, 35$ q, 2juv., (IEPA 00239); Vigia, 06.viii.1995, J. Roberto, 1 oov., (MPEG 71); Igarapé Anuerá, Vigia $\left(00^{\circ} 51^{\prime} 31^{\prime \prime} \mathrm{S} 48^{\circ} 08^{\prime} 31^{\prime \prime} \mathrm{W}\right), 10 . i i i .1995$, R.

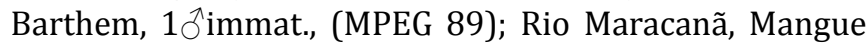
do Guilherme (left bank), Santarém-Novo, 25.iv.1998, F.R. Pimentel and R.N. Yuki, 1q, (MPEG 568); Acarajó, mouth of Rio Caeté, Bragança, 28.vi.1996, V.J. Isaac and A. Montenegro, $1 \hat{0}, 5$ immat., 3 o ov., 5juv., (MPEG 179); Furo Taici, Bragança, 15.vi.1997, M.P. de Barros and L.M.A. da Silva, 1 immat., (MPEG 269); Cachoeira do Arari $\left(01^{\circ} 00^{\prime} 52^{\prime \prime} \mathrm{S} 48^{\circ} 57^{\prime} 49^{\prime \prime} \mathrm{W}\right)$, mouth of Rio Goiapi, Ilha de Marajó, 08.ix.1990, I. Assunção, 1 immat., 19 ov., (MPEG 81); Baía de Marajó, mouth of Rio Arari, 05.vii.1984, R. Barthem, 1 , 12 ìmmat., 9 immat., 1 ov., (INPA 1099); Ilha de Mosqueiro, Igarapé Cajueiro, 19.v.1994, R. Barthem and R. Aragão, 1ठ̃, 2 ov., 1juv., (INPA 1216); Igarapé Porto da Seringa, Ananindeua $\left(01^{\circ} 21^{\prime} 57^{\prime \prime}\right.$ S 48 $22^{\prime} 21^{\prime \prime}$ W), 02.xi.1984, P. Sá and J. Ivan, $23^{\top}$ immat., (MPEG 90); Igarapé do Paracuri, Icoaraci $\left(01^{\circ} 18^{\prime} 07^{\prime \prime} \mathrm{S} 48^{\circ} 29^{\prime} 06^{\prime \prime} \mathrm{W}\right)$, 23.xii.1995, R.A.F. Maia, L.M.A. da Silva and M. Mourão Jr., 19ð̂, 13 + 5 @immat., (MPEG 35); idem, 24.viii.1995, R.A.F. Maia and L.A. Filgueiras, $4{ }^{1}$ immat., 4 qimmat., (MPEG 38); idem, 24.vi.1995, R.A.F. Maia and M. Mourão Jr., 1 + immat.,

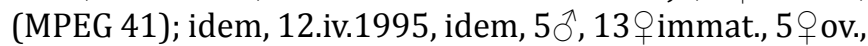
(MPEG 42); idem, 06.xi.1995, M. Mourão Jr. and M. Soares, $16 \hat{\jmath}, 11$, (MPEG 44); idem, 09.vii.1995, R.A.F. Maia and M. Mourão Jr., 5 immat., 2 ov., (MPEG 53); idem, 18.xii.1994, idem, $10{ }^{\lambda}$ (com 1 parasitado), 7 immat., $3{ }_{+}$ov., (MPEG 56); idem, 21.xii.1994, idem, $14 \hat{\jmath}, 2 \bigcirc, 9+$ immat., $4 \bigcirc$ ov., (MPEG 58); idem, 23.xii.1994, idem, 21 $\delta^{\lambda}, 19 \circ, 3$ ov., (MPEG 61); Igarapé do Paracuri, Icoaraci $\left(01^{\circ} 18^{\prime} 07^{\prime \prime} \mathrm{S}\right.$ $48^{\circ} 29^{\prime} 06^{\prime \prime}$ W), 10.ix.1995, R.A.F. Maia and M. Mourão Jr., 5 îmmat., 2 qimmat., (MPEG 335); idem, 26.v.1995, idem,
10̂, 1 immat., 2 ovo., (MPEG 338); Igarapé Sapucajuba (POEMA), Belém, 24.xii.1995, R.A.F. Maia and M. Mourão Jr., 8ð̄, 6ᄋ, (MPEG 2); idem, 10.viii.1995, coll. unknown, 16万人, 8ㅇ, (MPEG 11); idem, 27.xii.1995, R.A.F. Maia and

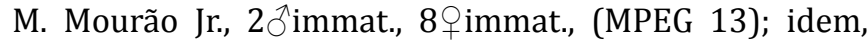
13.vii.1995, idem, $8 \hat{\jmath}, 3+, 6 q$ ov., (MPEG 16); Igarapé Sapucajuba (POEMA), Belém, 22.xi.1994, R.A.F. Maia and M. Mourão Jr., $5 \bigcirc$, 3 \% , (MPEG 67); idem, 31.iii.1995, idem,

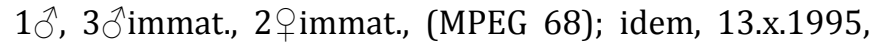

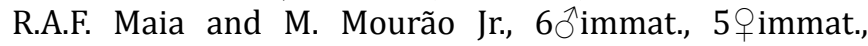
(MPEG 161); idem, 18.vii.1995, R.A.F. Maia and M. Mourão Jr., 2 immat., (MPEG 322); idem, 19.vii.1995, idem, 3ð̂, 3 , (MPEG 321); Igarapé Puraquequara, between Ourém and São Miguel do Guamá $\left(01^{\circ} 37^{\prime} 37^{\prime \prime}\right.$ S 47 $29^{\prime} 01^{\prime \prime}$ W), 13.xii.1995, A. Bezerra, J. Carvalho Jr., M. Aires, B.E. da Silva Barbosa and H. Higuchi, $3{ }^{\wedge}$ immat., 3 immat., (MPEG 134); Ilha de Marajó, Muaná, Furo do Palheta, 08.xi.1997, B. Mascarenhas and J.O. Dias, 1ð̄, (MPEG 526); Rio Buiaçu, Ilha de Marajó, Breves, 11.vi.1997, R.B. Neto, $2 \hat{\jmath}, 1+$ ov., (MPEG 257); Rio Ipiranga, community of Ilha da Salvação, Melgaço, 24.vi.1997, J.O. Dias, 10, (MPEG 262); bank at Ilha das Araras, Curralinho, 24.vii.1984, R. Barthem, $5 \hat{\jmath}, 1$ ㅇ, 6qov., (INPA 092); Rio Pará, Ilha das Araras, Curralinho, 25.vii.1984, R. Barthem, 1 qov., (INPA 1097); Santarém, iv.1999, coll. unknown, 20 , (INPA 1100); Banco das Araras, Curralinho, 24.vii.1984, R. Barthem, 4ત, 12 , 35 ov., (INPA 1102); Limoeiro do Ajuru, Ilha de Marajó, 18.x.1997, B. Mascarenhas, D. Guimarães and J.O. Dias, 11 đ 1ㅇ, (MPEG 520); Santarém, iv.1999, coll. unknown, 2へ, 3 , (MPEG 662); Ilha do Combú, Acará, 24.x.2001, Adélia, 3 îimmat., 6 immat., 10juv., (MPEG 772); Rio Tocantins $\left(02^{\circ} 02^{\prime} \mathrm{S} 49^{\circ} 17^{\prime} \mathrm{W}\right)$, between Rio Bom Jardim (upriver tributary) and Rio Pindobal (downstream tributary), between Curucambala and Maiauta, 20.xi.1994, A. Zanatra et al., $1{ }^{\top}$ immat., 1 ov., (INPA 1091); Rio Tocantins, 20.vi.1988, coll. unknown, 1 q, 1 ov., (INPA 1068); idem, 30.iv.1987, U. Barbosa, 12 đ̂immat., 6 immat., (INPA 1230); idem, between Cametá and Tucuruí, iii.1985, 0. Odinetz-Collart, 11ð̂, 3 @immat., 1 o ov., (INPA 284); idem, Acaripucu, 30.iv.1988, U. Barbosa, 69 ov., (INPA 1218); idem, Icangui, 25.iii.1985, 0. Odinetz-Collart, 80 $\hat{~}, 44 \not$, 55 ov., (INPA 183); idem, Icangui, 01.v.1988, U. Barbosa, 4 ov., (INPA 1220); idem, 01.xii.1987, idem, $1 \delta^{\Uparrow}$ immat., 4juv., (INPA 1232); idem, Igarapé Vermelho, upriver from Itupiranga, 12.vii.1987, U. Barbosa, $5{ }^{\text {immat., } 12}$ immat., 5juv., (INPA 1235). All records are shown in Figure 7.

\section{Palaemon Weber, 1795}

\section{Palaemon carteri (Gordon, 1935)}

Palaemonetes carteri Gordon 1935: 324, fig. 12.

Palaemonetes (Palaemonetes) carteri - Kensley and Walker 1982: 11, figs. 13, 14.

Palaemonetes carteri - Holthuis 1966: 6; Rodríguez 1981: 47 [in list]; Ramos-Porto and Coelho 1998: 337 [in list]; Barros and Pimentel 2001: 20 [in list]; Vieira 2003: 60, fig.; Melo 2003: 382 [map]; Magalhães and Pereira 2007: 14 [in list].

Geographic distribution. Venezuela, Guyana, Suriname, French Guiana, Brazil (Amapá, Amazonas, Pará) (see Pileggi et al. 2013 for references). 


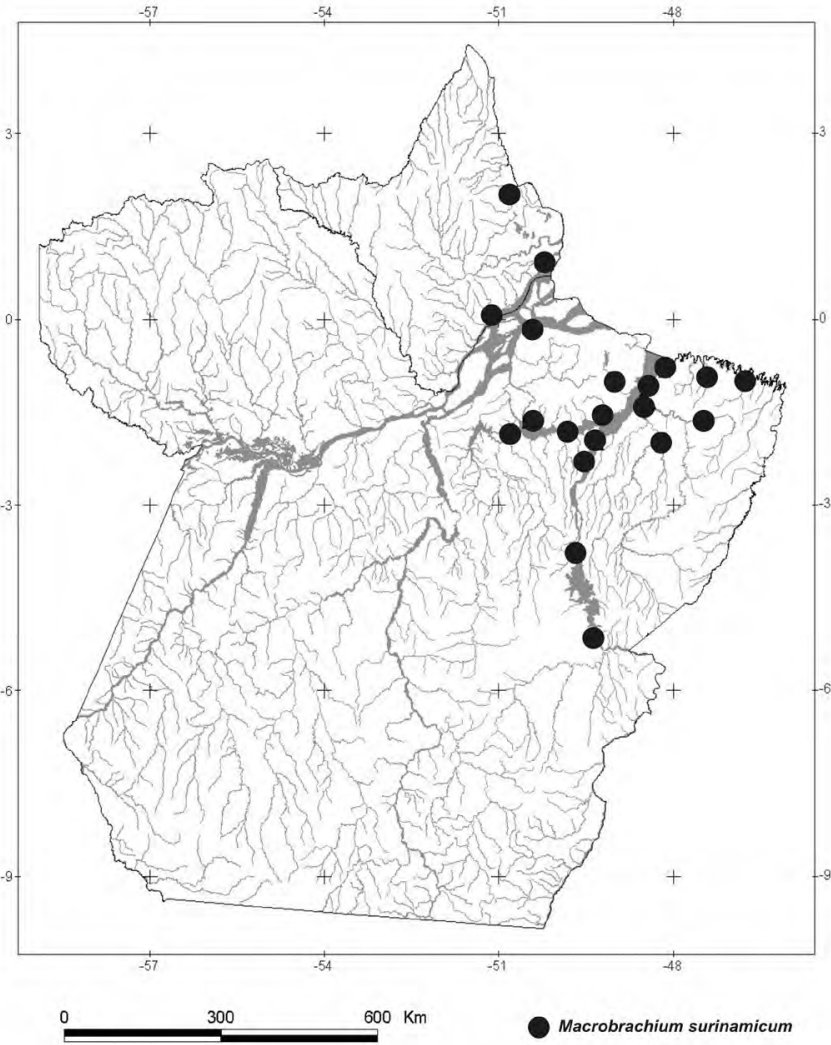

FIGURE 7. Geographic distribution of Macrobrachium surinamicum Holthuis, 1948 in the States of Amapá and Pará, Brazil (some symbols may represent more than one record)

Material examined. AMAPÁ: Rio Amapá Grande, Cachoeira Grande, small igarapé upstream from the fall, Amapá, 26.viii.1992, C. Magalhães and R. Pinto, $1 \delta^{\lambda}, 1$, (INPA 1083); Rio Araguari, Lago Piratuba Biological Reserve, channel to the lakes, date unknown, C. Magalhães, 2 immat., (INPA 1084); road Macapá-Jari, Igarapé do Parma. 20.iii.1991, V. Py-Daniel et al., 10̂, 2 + 3 ovo., (INPA 1208). PARÁ: Igarapé Bacuri, Santarém-Novo, 26.iv.1998, F.R. Pimentel and R.N. Yuki, 32 $̂$, 29ㅇ, (MPEG 563); Inajá, Igarapé Vilemar, Primavera, 07.ix.1987, B. Mascarenhas etal.,8 8 immat., 5 @immat.,(MPEG 782); Sítio Aracuã, Jequiri, between Bragança and Augusto-

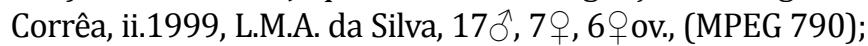
idem, 15.xi.1999, F.R. Pimentel and R. Souza, 36 $\hat{\circ}, 11$, 8 ov., (MPEG 787); Rio Piracatuba near Ilha do Mosqueiro, 29.x.1984, J. Porto and P. Rocha, $1 \delta, 1$ immat., 1 iov., (MPEG 779); bridge at road Ourém-São Miguel do Guamá, Igarapé Poraquequara, 13.xii.1995, A. Bezerra, J. Carvalho Jr., M. Aires, B.E. da Silva Barbosa and H. Higuchi, $7 ð, 3 \bigcirc$, (MPEG 133); road PA-140, Km 18, road to Bujaru, 24.ix.1997, E. Monteiro, 1ㅇ, (MPEG 464); Reserva Mocambo-APEG, Belém, 07.v.1997, R.N. Bittencourt, 1q, (MPEG 200); idem, 17.ix.1997, M.P. de Barros, F.R. Pimentel, S.S.B. da Silva and R.N. Bittencourt, $1 \widehat{\delta}$, 19, (MPEG 449); idem, 09.i.1998, F.R. Pimentel and R.A.F. Maia, 13 ${ }^{\lambda}, 12$, , (MPEG 528); idem, 14.vii.1998, F.R. Pimentel

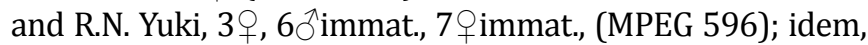
01.viii.1998, idem, 21ð̄, 12ᄋ, (MPEG 598); idem, 18.iii.1978,

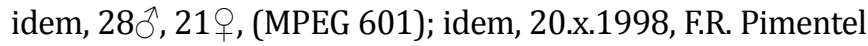
and D. Guimarães, 19 $\hat{\text {, }} 23$ + (MPEG 604); idem, 18.xi.1998, F.R. Pimentel and R.N. Yuki, 43ð, 37ᄋ, (MPEG 606); idem, 15.xii.1998, idem, 27ð̂, 24ㅇ, (MPEG 609); idem, 21.i.1999, F.R. Pimentel and R.M. dos Santos, 17 $\hat{\jmath}, 119$, (MPEG 612); idem, 24.ii.1999, F.R. Pimentel and J.O. Dias, $4 \hat{\circ}, 1+$, 8 ov., (MPEG 616); idem, 19.iii.1999, idem, 1ㅇ, $1 \delta^{\Uparrow}$ immat., (MPEG
619); idem, 22.iv.1999, F.R. Pimentel and R.M. dos Santos, $4 \hat{\jmath}, 2$, (MPEG 622); idem, 21.v.1999, F.R. Pimentel and J.O. Dias, 1 q , 8 immat., 5 immat., (MPEG 625); idem, 18.vi.1999, idem, 22 $\bigcirc, 19 \circ$, (MPEG 628); idem, 21.vii.1999,

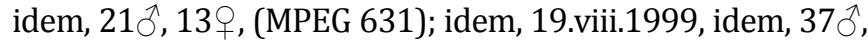
22ㅇ, (MPEG 634); idem, 30.ix.1999, idem, 16 637); idem, 21.x.1999, idem, 35ð, 31ㅇ․ (MPEG 640); idem, 23.xi.1999, idem, 37 $\bigcirc, 21$, (MPEG 642); idem, 22.xii.1999, idem, 39 $\hat{\jmath}, 27$, (MPEG 644); idem, 19.i.2000, idem, 7§, 3ㅇ, (MPEG 646); idem, 24.ii.2000, idem, 2ㅇ, 6ðึimmat., 1 ov., (MPEG 649); idem, 24.iii.2000, idem, 20, 1 (qmmat., 1juv., (MPEG 651); idem, 27.iv.2000, idem, 9ð, 11, (MPEG 653); idem, 25.v.2000, idem, 16へ, 13ㅇ, (MPEG 655); Km 03 (side road of the PA-252 road), Igarapé Jupuúba, road between Bujaru and Acará $\left(01^{\circ} 58^{\prime} 35,0^{\prime \prime} \mathrm{S} 48^{\circ} 08^{\prime} 32,5^{\prime \prime}\right.$ WO), 25.ix.1997, E. Monteiro, 1ठ․ (MPEG 463); Balneário Oriental II, Igarapé Jupuúba, Acará, 08.x.1997, G.J.A. Silva, 11ठ, 8ㅇ, (MPEG 516); Vila do Conde, Barcarena, 23.iii.2002, B. Mascarenhas, $16 \hat{\jmath}, 5$, 5 qov., (MPEG 739); idem, 24.iii.2002, B. Mascarenhas, 24 spec., (MPEG 741); Vila Arienga, Igarapé Arienga, Barcarena, 26.iii.2002, B. Mascarenhas, 2 spec., (MPEG 744); Vila do Conde, Linhão, Barcarena, 25.iii.2002, B. Mascarenhas et al., 196 spec., (MPEG 751); Rio Amazonas, São Raimundo, Almeirim, 12.v.1999, J. Carvalho Jr., 31 $\partial^{1}, 7$, (MPEG 685); idem, Pesqueiro, quadra 32, 26.vii.1999, idem, 59ð, 48 9 , 8 ov., (MPEG 689); Rio Araiollos, Rio Amazonas basin, Pesqueiro São Paulo, Almeirim, 27.vii.1999, idem, $6 \AA, 2$, (MPEG 691); ECFPn, FLONA Caxiuanã, Igarapé Poraquequara, Melgaço, 07.xi.1999, coll. unknown, 13ð, 9ᄋ, (MPEG 719); idem, 06.xi.1999, idem, 43ð, 31, (MPEG 722); community of Guajará, Santarém, 15.v.1999, coll. unknown, 1 ov., (MPEG 684); community of São João do Uricurituba, Santarém, 29.xii.1999, M.R. dos Reis, 2 + , (MPEG 696); Lago Jacundá, Alter-do-Chão, Santarém, 16.ii.2000, C.R. García-Dávila, 27 $, 29 q, 12$ ov., (INPA 1107); Lago Cavari, idem, 26ð̃, 25ㅇ, 18 ov., (INPA 1113); headwaters of Lago

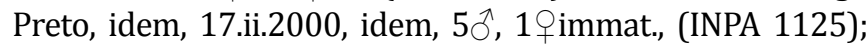
headwaters of Lago Verde, idem, 18.ii.2000, idem, 2ð, (INPA 1130); Lago Minitiapina, idem, 19.ii.2000, idem, $8 \hat{\jmath}, 4$, 19 ov., (INPA 1126); Lago Jucuruí, idem, 21.ii.2000, idem, $1 \overbrace{}^{\lambda}$, 2 ov., (INPA 1140); Lago Iruçanga, idem, 21.ii.2000, idem, $31 \hat{\jmath}, 30 \circ, 24 \bigcirc$ ov., (INPA 1143); Lago Jacaré, idem, $3{ }^{\lambda}$, 8 , 2 îmmat., 1 immat., 1 ov., (INPA 1147); Lago Verde, idem, 18.ii.2000, idem, $11 \hat{\jmath}, 8 \bigcirc, 2$ ov., (INPA 1137); Igarapé do Irurama, idem, 17.ii.2000, idem, 10̂, 1 ov., (INPA 1116); Lago das Mangueiras, idem, 21.ii.2000, idem, $9 \hat{\jmath}$, (INPA 1149); Rio Xingu, Ilha do Forno, Altamira, 18.xii.2000, R. Souza and Dionísio, 4ㅇ, (MPEG 715); left bank of Rio Tapajós, near mouth of Rio Cupari, 27.x.1991, C. Magalhães and L. Rapp Py-Daniel, 12خ, 28ㅇ, (INPA 1176); idem, between Buburé and São Luis, 22.x.1991, J. Zuanon and equipe - INPA, 17 $\hat{\jmath}, 22$, (INPA 1177); Rio Cupari, near mouth, 27.x.1991, A. Negrão et al., 3ㅇ, (INPA 1199); Rio Tapajós, igarapé on the left bank, near Rio Cupari, 26.x.1991, C. Magalhães and J. Zuanon, 1ठ, 1 , (INPA 1200); Rio Tapajós, near mouth of Rio Cupari, 26.x.1991, C. Magalhães, J. Zuanon and E.N. Santos Silva, 2 , (INPA 1201); left bank of Rio Tapajós downstream from Buburé, 22.x.1991, C. Magalhães, 3ㅇ, (INPA 1206); Rio Xingu, Paraná do Maxacá, 19.x.1992, M. Jegu, 7 îmmat., 10 immat., 1 ovo., (INPA 1159); idem, Lago do Poção, Igarapé do Poção, 11.x.1992, V. Py-Daniel et al., 
4 îmmat., 9 ○immat., (INPA 1171); Rio Trombetas, Lago Jamari, 23.ii.1982, coll. unknown, 15ð, 77우, (INPA 1101); Rio Trombetas (right bank), Lago Tapagem, 11.x.1985, C. Magalhães, 19ð, 95ㅇ, (INPA 336); Balneário Atay, Monte Dourado, 09.iv.2002, B. Mascarenhas et al., 2 spec., (MPEG 747). All records are shown in Figure 8.

Palaemon mercedae (Pereira, 1986)

Palaemonetes mercedae Pereira 1986: 209, fig. 13.

Geographic distribution. Venezuela, Colombia, and Brazil (Amazonas, Pará) (see Pileggi et al. 2013 for references).

Material examined. PARÁ: Santarém, Alter do Chão, Igarapé do Irurama, 17.ii.2000, C.R. García-Dávila, $1 \widehat{ }$ 1, (INPA 1116); Santarém, Alter-do-Chão, Igarapé São Sebastião, 17.ii.2000, C.R. García-Dávila, 2 $\hat{\circ}, 3$, $2+$ ov., (INPA 1119). All records are shown in Figure 8.

Palaemon pandaliformis (Stimpson, 1871)

Leander pandaliformis Stimpson 1871: 130.

Palaemon pandaliformis - Barros and Pimentel 2001: 20 [in list].

Geographic distribution. Antilles, Central and South America, in Brazil (Pará, from Rio Grande do Norte to Rio Grande do Sul) (Holthuis 1952; Villalobos-Figueiroa 1982; Delgado et al.1997; Coelho and Ramos-Porto 1998; Barros and Pimentel 2001).

Material examined. PARÁ: Furo Taici, Bragança, 25.iv.1997, M.P. de Barros and L.M.A. da Silva, 38 $\overbrace{}^{1}, 25 ㅇ$,

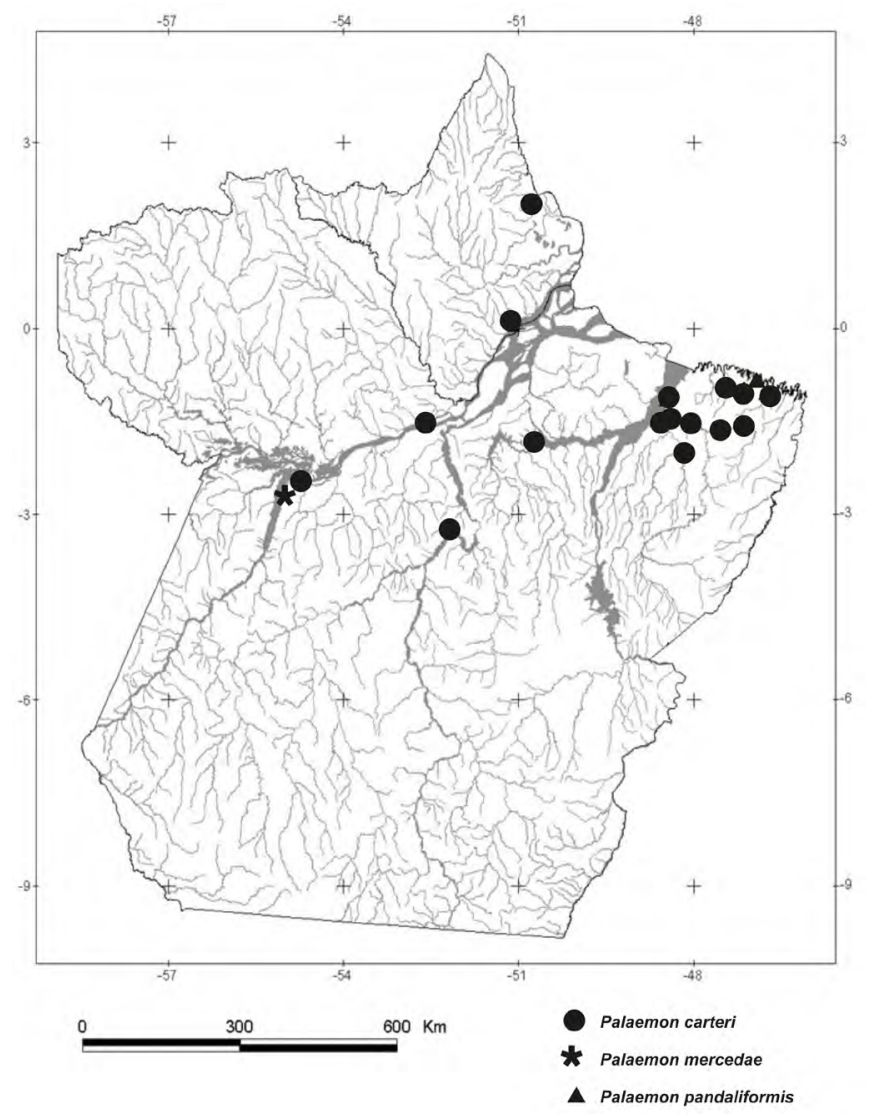

FIGURE 8. Geographic distribution of Palaemon carteri (Gordon, 1935), P. mercedae (Pereira, 1986), and P. pandaliformis (Stimpson, 1871), in the States of Amapá and Pará, Brazil (some symbols may represent more than one record).
(MPEG 195); idem, 07.ii.1997, M.P. de Barros and R.V. do Espírito Santo, 1ㅇ, (MPEG 205); idem, 15.vi.1997, M.P. de Barros and L.M.A. da Silva, 82 $\bigcirc, 53$, (MPEG 267); idem, 12.vi.1997, M.P. de Barros and R.V. do Espírito Santo, 20 ऽ, 11오 (MPEG 282); idem, 09.viii.1997, M.P. de Barros and A. Montenegro, 76 ${ }^{\lambda}, 41$, , (MPEG 430); idem, 27.ix.1997, idem, 81ð, 37@, (MPEG 468). All records are shown in Figure 9.

Pseudopalaemon Sollaud, 1911

Pseudopalaemon amazonensis Ramos-Porto, 1979 Pseudopalaemon amazonensis Ramos-Porto 1979: 693.

Pseudopalaemon amazoniensis [sic] - Barros and Pimentel 2001: 20 [in list]

Geographic distribution. Venezuela and Brazil (Amazonas, Pará) (Pereira 1991; Kensley and Walker 1982).

Material examined. PARÁ: ECFPn, FLONA de Caxiuanã,

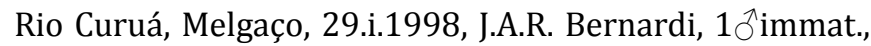
1 immat., (MPEG 536); road PA-140, Km 30, Jundiaí, between Santa Isabel and Bujaru, E. Monteiro, 10, 1 immat., 1juv., (MPEG 451). All records are shown in Figure 9.

Pseudopalaemon chryseus Kensley \& Walker, 1982

Pseudopalaemon chryseus Kensley and Walker 1982: 16, figs. 18-19; Magalhães and Pereira 2007: 14 [in list];

Vieira 2008: 69 [in list]; Pileggi et al. 2013: 570.

Pseudopalaemon cf. chryseus - Vieira 2003: 61, fig.

Geographic distribution. Colombia, Brazil (Amapá, Amazonas, Pará) (see Pileggi et al. 2013 for references).

Material examined. AMAPÁ: road Macapá-Jari, Igarapé do Parma, V. Py-Daniel et al., 20.iii.1991, coll. unknown, 10̂, (INPA 1215). PARÁ: lower Rio Trombetas basin, unnamed lake at right bank, $3 \mathrm{~km}$ upstream from Juquiri, 07-08.iii.1986, V. Py-Daniel et al., 3ㅅ, 2 ㅇ, (INPA 452); Rio Curuá-Una, i-ii.1981, R. Best, $1 \hat{\sigma}, 1$ imat, $3 q$ ov., (INPA 073); Rio Cupari, near mouth of Rio Tapajós, 27.x.1991., A. Negrão et al., 2 imat, 1 , (INPA 1186); Rio Tapajós, Itaituba, 18.x.1991, C. Magalhães et al., 12q, 5juv., (INPA 1189); left bank of Rio Tapajós near mouth of Rio Cupari, 27.x.1991, C. Magalhães and L. Rapp Py-Daniel, 3 imat, (INPA 1204); Rio Trombetas, Cachoeira Porteira, 05.vi.1986, U. Barbosa, 1 , , (INPA 1085); Rio Xingu, Paraná do Maxacá, 19.x.1992, M. Jegu, 32ð̂, 1 \%, 43 †immat., (INPA 1160); idem, Ilha de Babaquara, 05.x.1990, J. Zuanon, 102 immat., (INPA 1161); Lago Jacundá, idem, 16.ii.2000,

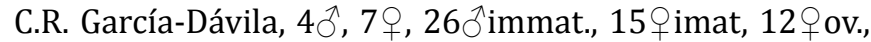
(INPA 1106); headwaters of Lago Preto, idem, 17.ii.2000,

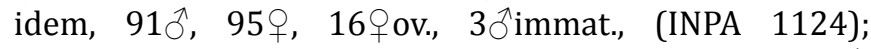
headwaters of Lago Verde, idem, 18.ii.2000, idem, $7 \hat{\jmath}$, $9 \bigcirc, 4$ ov., (INPA 1129); Lago Piranha, idem, 16.ii.2000, idem, $24 \hat{\jmath}, 31$ ㅇ, 2 ov., (INPA 1139); Lago Verde, idem, 18.ii.2000, idem, 1ㅇ, (INPA 1133); Lago Caravari, idem, 16.ii.2000, idem, 2 q $10 \hat{\jmath}$, (INPA 1114); Lago Iruçanga, idem, 21.ii.2000, idem, 2 ㅇ, $5 \hat{\jmath}, 1$ ov., (INPA 1142); Lago Jacaré, idem, 21.ii.2000, idem, 8 immat., $4 \hat{\jmath}$, (INPA 1146); ECFPn, FLONA Caxiuanã, Rio Curuá, Melgaço, 08.xi.1999, coll. unknown, $5 \hat{\jmath}, 2$, 4 immat., 1 oov., (MPEG 718); idem, Igarapé Poraquequara, Melgaço, 10.xi.1999, coll. 
unknown, 1̧ิ, 5 ov., (MPEG 726); Balneário Atay, Monte Dourado, 09.iv.2002, B. Mascarenhas et al., 3+, (MPEG 746); Reserva Genética Felipe, Monte Dourado, 14.iv.2002, B. Mascarenhas et al., 13 spec., (MPEG 749); ECFPn, FLONA Caxiuanã, Rio Curuá, Melgaço, 18.iii.2001, coll. unknown, 12 immat., $1 \delta, 3$, 2 ov., 14juv., (MPEG 765); idem, Rio Caxiuanã, Melgaço, 19.iii.2001, coll. unknown, 2ð, 13 i immat., 26juv., (MPEG 767); idem, mouth of Rio Curuá, Melgaço, xi.1999, coll. unknown, 1 ov., (MPEG 780). All records are shown in Figure 9.

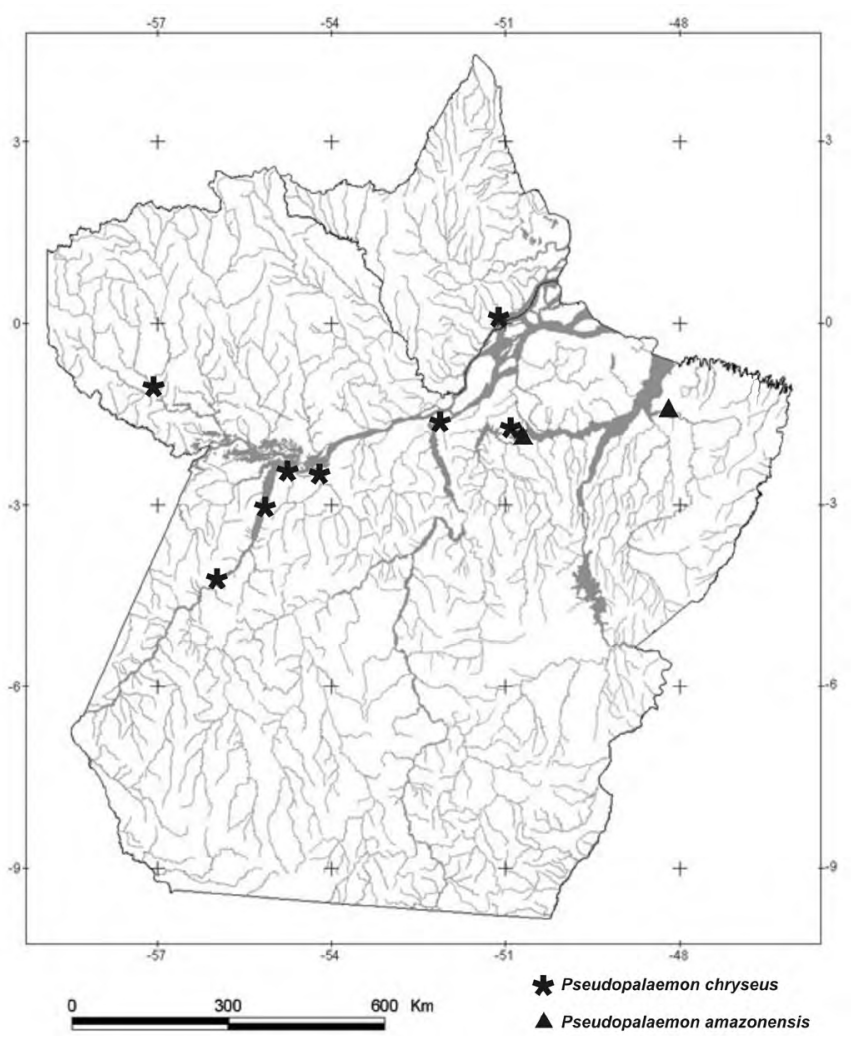

FIGURE 9. Geographic distribution of Pseudopalaemon amazonensis Ramos-Porto, 1979, and Ps. chryseus Kensley and Walker, 1982 in the States of Amapá and Pará, Brazil (some symbols may represent more than one record).

\section{Discussion}

The distribution of freshwater shrimps from Amapá and Pará is well known, but its diversity is likely to be underestimated, since faunistic surveys are scarce and vast areas are still poorly explored. For this same reason, the distribution of many species might be larger then informed by the records presented in this paper (Figures 1-9).

Macrobrachium brasiliense is widespread, occurring in coastal rivers of Amapá and in most of the State of Pará. $M$. nattereri has also a wide distribution, occurring from the western to the eastern part of the State of Pará; the species was not recorded in the State of Amapá. Macrobrachium amazonicum and $M$. surinamicum have roughly the same distribution pattern, both occuring in the eastern and southeastern portions of the States of Amapá and Pará, as well as in the northeast portion of Pará, with records in the Pará River and the Tocantins and Guama Rivers. The distribution of $M$. amazonicum also covers the western portion of the State of Pará, occurring in the Xingu River. The distribution of the $M$. jelskii is similar to that of $M$. amazonicum, with the records being distributed mainly along the Amazon River, as well as in the coastal river basins of Amapá and Pará.

Acetes marinus, Macrobrachium carcinus, M. acanthurus, M. olfersii and Palaemon pandaliformis have a more restricted, almost punctual distributions, which cover coastal basins of the States of Amapá and Pará. M. olfersii is widely distributed in the Americas (Bowles 2000) and the data obtained in this work confirm its occurrence in the State of Amapá. However, its occurrence in Pará could not be confirmed, probably due to lack of collections. $M$. carcinus occurs in the lower portion of both the Amazonas and Tocantins rivers.

Palaemon mercedae, Pseudopalaemon chryseus, $P$. amazonensis, and the three species of Euryrhynchus occur in less extensive areas, usually associated with submerged leaf litter habitat of the acidic, nutrient-poor, black and clear water river systems that drain the Guyana and central Brazil shields.

The genus Euryrhynchus has three representatives in the Amazon basin: E. amazoniensis, E. burchelli, and E. wrzesniowskii. The first species was thought to be restricted to the central Amazon region, but García-Dávila and Magalhães (2004) extended the range to the western Amazon, in the Peruvian province of Requena, and Vieira (2003; 2008) recorded it from the State of Amapá. E. burchelli, previously reported from Pará by Tiefenbacher (1978) and Holthuis (1951; 1966), is known from the west and northeast portions of this state; the species was also reported from the eastern Amapá (Vieira 2003). The distribution of E. wrzesniowskii encompasses Guyana and Suriname (Tiefenbacher 1978), and the central (Kensley and Walker 1982), and eastern (Vieira 2003; 2008) Amazon basin. In the present study, its distribution range is extended to northeastern Pará.

Three species of the genus Palaemon are found in the inland waters of the Amazon basin: Palaemon ivonicus, P. carteri, and P. mercedae, but only the last two have been recorded in the eastern Amazon region. In Amapá, $P$. carteri is distributed in the northeast and southeast parts of the State of Amapá, while in the State of Pará it ranges from the western portion, in the Trombetas River, to the northeastern portion of the state. P. mercedae was described from Venezuela (Pereira 1986) and has subsequently been found in Central Amazon (Magalhães 1988). Pileggi et al. (2013) firstly reported the occurence of this species in Pará, in the Tapajós River Basin.

The genus Pseudopalemon is endemic to South America (Kensley and Walker 1982) and six species are known from the Amazon basin, but only two have been found in eastern Amazon. Barros and Pimentel (2001) listed $P$. amazonensis for Pará, but did not offer documented data on its occurrence. The records presented herein show that the species is found from the Caxiuanã Bay to northeastern Pará, inhabiting black-water forest streams. P. chryseus has been recorded from the southeastern portion of Amapá (Vieira 2003; Pileggi et al. 2013), and from Trombetas River to the lower Amazon basin, in Pará (Pileggi et al. 2013).

The two freshwater species of Sergestidae occur in eastern Amazon region. A. marinus is mainly found in areas influenced by oligohaline waters and its range is 
restricted to the lower Amazon and Tocantins Rivers, and coastal rivers of the State of Amapá (Omori 1975; D'Incao and Martins 2000; present paper). A. paraguayensis has a very wide distribution in South America, ranging from the Orinoco and Amazon basins to the lower Paraná River basin (Pileggi et al. 2013). It occurs in the Amazon River and its main tributaries in Pará (Trombetas, Tapajós, Xingu, Tocantins, and Pará Rivers), but has not yet been recorded in Amapá.

Additionally, two exotic species from the Indo-Pacific region have also been reported from Pará. Barros and Silva (1997) recorded Macrobrachium rosenbergii (De Man, 1879) from the surroundings of Belém and Bragança. A study using molecular analysis made by Iketani et al. (2011) determined that the species introduced in the coastal areas of Pará was actually M. dacqueti (Sunier, 1925), but the name $M$. rosenbergii must be kept (Wovor and Ng 2008; ICZN 2010). Maciel et al. (2011) found Macrobrachium equidens (Dana, 1852) from the Taici Creek, a tidal channel that connects the Caeté and Taperaçu Rivers, near Brangança, northeastern Pará.

ACKNowledgments: The authors wishes to thank the Conselho Nacional de Desenvolvimento Científico e Tecnológico - CNPq for a Master Degree Fellowship to FRP, and for an ongoing Research Grant to CM (Proc. 303837/2012-6); the curators of the invertebrate collections at IEPA, I. M. Vieira, and MPEG, A.B. Bonaldo and C.R.M. dos Santos, for their assistance; and B. Robertson and Diogo Provete, for correcting the English text. We also thank G. Bond-Buckup for her comments to an earlier version of the manuscript, and two anonymous reviewers for suggestions that greatly improved the manuscript.

\section{Literature Cited}

Barros, M.P. and F.R. Pimentel. 2001. Decapoda (Crustacea) do estado do Pará, Brasil: lista preliminar das espécies. Belém, Boletim do Museu Paraense Emílio Goeldi, série Zoologia 17(1): 15-41.

Barros, M.P. and L.M.A. Silva. 1997. Registro de introdução da espécie exótica Macrobrachium rosenbergii (De Man, 1879) (Crustacea, Decapoda, Palaemonidae), em águas do Estado do Pará, Brasil. Belém. Boletim do Museu Paraense Emílio Goeldi, série Zoologia 13(1): 31-37.

Bentes, B. S., J.M. Martinelli, L.S. Souza, D.V. Cavalcante, M.C. Almeida and V.J. Isaac. 2011. Spatial distribution of the Amazon River prawn Macrobrachium amazonicum (Heller, 1862) (Decapoda, Caridea, Palaemonidae) in two perennial creeks of an estuary on the northern coast of Brazil (Guajará Bay, Belém, Pará). Brazilian Journal of Biology 71(4): 925-935. (http://www.scielo.br/pdf/bjb/v71n4/13.pdf)

Bond-Buckup, G. and L. Buckup. 1989. Os Palaemonidae de águas Continentais do Brasil Meridional (Crustacea, Decapoda). Revista brasileira de Biologia 49(4): 883-896.

Bowles, D.E., K. Azis and C.L. Knight. 2000. Macrobrachium (Decapoda: Caridea: Palaemonidae) in the contiguous United States: A review of the species and na assessment of threats to their survival. Journal of Crustacean Biology 20(1): 158-171. (doi: 10.1163/2002197599990025)

Calman, W.T. 1907. On a Freswater Decapod Crustacean Collected by W.J. Burchell at Pará in 1829. Annals and Magazine of Natural History, série 7, 19: 295-299.

Cavalcante, D.V., B.B. Silva and J.M. Martinelli-Lemos. 2012. Biodiversity of decapod crustaceans in the estuarine floodplain around the city of Belém (Pará) in Brazilian Amazonia. Zoologia 29(3): 203-209. (doi: 10.1590/S1984-46702012000300003)

Coelho, P.A. and M. Ramos-Porto. 1985. Camarões de água doce do Brasil: Distribuição Geográfica. Revista brasileira de Biologia 2(6): 405-410.

D'Incao, F. 1998. Malacostraca-Eucarida-Dendrobranchiata; pp. 311-321, in: P.S. Young. (ed.). Catalogue of Crustacea of Brazil. Rio de Janeiro: Museu Nacional, xvii+717p. (Série Livros 6)

D'Incao, F. and S.T.S. Martins. 2000. Brazilian species of the genera Acetes H. Milne-Edwards, 1830 and Pleisos Burkenroad, 1945 (Decapoda: Sergestidae). Journal of Crustacean Biology 20(2): 78-86. (http:// links.jstor.org/sici?sici=0278-0372\%28200006\%2920\%3A2\%3C78 \%3ABSOTGA\%3E2.0.CO\%3B2-6)

De Grave, S. 2007. A new species of Euryrhynchus Miers, with a discussion of the systematic position of the Euryrhynchidae Holthuis (Crustacea, Decapoda). Zoologischer Anzeiger 246: 193-203. (doi:10.1016/j. jcz.2007.06.002)
Delgado, J.G., H.J. Severeyn, A.R. Godoy, Y.M.Reverol and J.J. Ewald, 1997. Camarones dulceacuícolas y estuarinos de Venezuela (Atyidae, Palaemonidae): nuevos registros para los estados Zulia y Falcón. Boletin del Centro de Investigaciones Biológicas 31(1): 11-32.

Ferreira, R.S., R.R.R. Vieira and F. D'Incao. 2010. The marine and estuarine shrimps of the Palaemoninae (Crustacea: Decapoda: Caridea) from Brazil. Zootaxa 2606: 1-24. (http://www.mapress.com/ zootaxa/2010/f/zt02606p024.pdf)

Freire, J. L.; C. Marques and B. Bentes. 2012a. Estrutura populacional e biologia reprodutiva de Macrobrachium amazonicum (Heller, 1862) (Decapoda: Palaemonidae) em um estuário da região Nordeste do Pará. Brazilian Journal of Aquatic Science and Technology 16(2): 65-76. (http://siaiweb06.univali.br/seer/index.php/bjast/article/ view/3427/2470)

Freire, J. L.; C. Marques and B. Bentes. 2012b. Crescimento e avaliação do estoque de Macrobrachium amazonicum em um estuário do Nordeste do Pará, Brasil. Boletim do Instituto de Pesca 38: 215-229. (ftp://ftp. sp.gov.br/ftppesca/38_3_215-229.pdf)

García-Dávila, C.R. and C. Magalhães. 2004. Revisão taxonômica dos camarões de água doce (Crustacea: Decapoda: Palaemonidae, Sergestidae) da Amazônia peruana. Acta Amazonica 33(4): 663-686 [2003]. (doi: 10.1590/S0044-59672003000400013)

Gordon, I. 1935. On new or imperfectly known species of Crustacea Macrura. Journal of the Linnean Society, Zoology 39: 307-351.

Heller, C. 1862. Beiträge zur näheren Kenntniss der Macrouren. Sitzungsberichte der Mathematisch-Naturwissenschaftlichen Klasse der Kaiserlichen Akademie der Wissenschaften, Wien 45(1): 389-426, pls. $1-2$.

Holthuis, L.B. 1948. Note on some Crustacea Decapoda Natantia from Surinam. Proceedings Koninklijke Nederlandsche Akademie van Wetenschappen 51(9): 1104-1113.

Holthuis, L.B. 1951. A general revision of the Palaemonidae (Crustacea, Decapoda, Natantia) of the Americas I. The subfamilies Euryrhynchynae and Pontoniinae. Allan Hancock Foundation Publications, Occasional Papers 11: 1-332.

Holthuis, L.B. 1952. General revision of the Palaemonidae (Crustacea: Decapoda: Natantia) of the Americas II. The Subfamily Palaemoninae. Los Angeles, Allan Hancock Foundation Publications, Occasional Papers 12: 1-396.

Holthuis, L.B. 1966. A collection of freshwater prawns (CrustaceaDecapoda-Palaemonidae) Brazil, collected by Dr. G. Marlier. Bulletin de l'Institut Royal des Sciences Naturelles de Belgique 42(10): 1-11.

ICZN. 2010. OPINION 2253 (Case 3428) Palaemon rosenbergii De Man, 1879 (currently Macrobrachium rosenbergii; Crustacea, Decapoda): usage conserved by designation of a neotype. Bulletin of Zoological Nomenclature 67(3): 258-260. (http://iczn.org/content/opinion2253-case-3428-palaemon-rosenbergii-de-man-1879-currentlymacrobrachium-rosenbergii-)

Iketani, G., L. Pimentel, G.C.O. da Silva, C.R. Maciel, W.C. Valenti, H. Schneider and I. Sampaio. 2011. The history of the introduction of the giant river prawn, Macrobrachium cf. rosenbergii (Decapoda, Palaemonidae), in Brazil: New insights from molecular data. Genetics and Molecular Biology 34(1): 142-151. (http://www.scielo.br/pdf/ gmb/v34n1/2010-107.pdf)

Kensley, B. and Walker, I. 1982. Palaemonid shrimps from the Amazon basin, Brazil (Crustacea: Decapoda: Natantia). Smithsonian Contribution to Zoology 362: 1-28. (doi: 10.5479/si.00810282.362)

Linnaeus, C. 1758. Systema Naturae per Regna Tria Naturae, Secundum Classes, Ordines, Genera, Species, cum Characteribus, Differentiis, Synonymis, Locis. (10 Edition) Vol. 1. Holmiae: Laurentii Salvii. iii, 824 pp. (doi: 10.5962 /bhl.title.542)

Lucena-Frédou, F; J.S. Rosa Filho, M.C.N. Silva and E.F. Azevedo. 2010. Population dynamics of the river prawn, Macrobrachium amazonicum (Heller, 1862) (Decapoda, Palaemonidae) on Combu island (Amazon estuary). Crustaceana 83(3): 277-290. (doi: 10.1163/001121609X1 2596543952298)

Maciel, C.R.; M. Quadros, F. Abrunhosa, S. Bastos, H. Schneider and I Sampaio. 2011. Occurrence of the Indo-Pacific freshwater prawn Macrobrachium equidens Dana 1852 (Decapoda, Palaemonidae) on the coast of Brazilian Amazonia, with notes on its reproductive biology. Anais da Academia Brasileira de Ciências 83: 533-544. (http://www.scielo.br/pdf/aabc/v83n2/v83n2a13.pdf)

Magalhães, C. 1988. The Development of Palaemonid Shrimps from the Amazon region reared in the laboratory. III. Extremely abbreviated larval development of Palaemonetes (Palaemonetes) mercedae Pereira, 1986 (Crustacea: Decapoda). Studies Neotropical Fauna Environment 23(1): 1-8. (doi: 10.1080/01650528809360739)

Magalhães, C. and G. Pereira. 2007. Assessment of the decapod crustacean diversity in the Guayana Shield region aiming at conservation decisions. Biota Neotropica, 7(2): 111-124. (http://www.scielo.br/ pdf/bn/v7n2/a13v07n2.pdf)

Martin, J.W. and G.E. Davis. 2001. Un Updated classification of the Recent 
Crustacea. An updated classification of the Recent Crustacea. Natural History Museum of Los Angeles County, Science Series 39: 1-124. (http://web.vims.edu/tcs/LACM-39-01-final.pdf)

Melo, G.A.S. 2003. Famílias Atyidae, Palaemonidae e Sergestidae; pp. 289-415, in: G.A.S. Melo (ed). Manual de Identificação dos Crustáceos Decápodos de Água Doce do Brasil. São Paulo: Editora Loyola.

Miers, E.J. 1877. On a collection of Crustacea, Decapoda and Isopoda, chiefly from South America, with descriptions of new genera and species. Proceedings of the Scientific Meetings of the Zoological Society of London 1877: 653-679, pls. 66-69.

Odinetz-Collart, O. 1987. La pêche crevettière de Macrobrachium amazonicum (Palaemonidae) dans le Bas Tocantins, après la fermeture du barrage de Tucuruí. Revue d'Hydrobiolgie Tropicale 20(2): 131-144.

Odinetz-Collart, 0. 1991. Tucurui dam and the populations of the prawn Macrobrachium amazonicum in the lower Tocantins (PA-Brasil): a four years study. Archiv für Hydrobiologie 122(2): 213-227.

Omori, M. 1975. The sistemactics, biogeography, and fishery of epipelagic shrimps of the genus Acetes (Crustacea, Decapoda, Sergestidae). Bulletin of the Ocean Research Institute, University of Tokyo 7: 54-59.

Pereira, G.A. 1986. Freshwater shrimps from Venezuela I: seven new species of Palaemoninae (Crustacea: Decapoda: Palaemonidae). Proceeding of the Biological Society of Washington 99(2): 198-213. (http://www.biodiversitylibrary.org/page/34595665\#page/222/ mode/1up)

Pereira, G.A. 1991. Camarones de agua dulce de Venezuela II: Nuevas adiciones en las familias Atyidae y Palaemonidae (Crustacea, Decapoda, Caridea). Acta Biologica Venezolana 13(1-2): 75-88.

Pileggi, L. G. and F.L. Mantelatto. 2010. Molecular phylogeny of the freshwater prawn genus Macrobrachium (Decapoda, Palaemonidae), with emphasis on the relationships among selected American species. Invertebrate Systematics 24(2): 194-208. (doi : 10.1071/ IS09043)

Pileggi, L.G., C. Magalhães, G. Bond-Buckup and F.L. Mantelatto. 2013. New records and extension of the known distribution of some freshwater shrimps in Brazil. Revista Mexicana de Biodiversidad 84(2): 563-574. (doi: 10.7550/rmb.30504)

Ramos-Porto, M. 1979. Pseudopalaemon amazonensis, espécie nova de camarão da bacia Amazônica (Crustacea, Decapoda, Palaemonidae). Sociedade Brasileira para o Progresso da Ciência, 31 ${ }^{\text {a }}$ Reunião Anual, Resumos. Ciência e Cultura, Suplemento 31(7): 693.

Ramos-Porto, M. and P.A. Coelho. 1990. Sinopse dos crustáceos decápodos brasileiros (Família Palaemonidae). Anais da Sociedade Nordestina de Zoologia 3(3): 93-111.

Ramos-Porto, M. and P.A. Coelho. 1998. Malacostraca-Eucarida-Caridea (Alpheoidea excluded); pp. 325-350, in: P.S. Young (ed.). Catalogue of Crustacea of Brazil. Rio de Janeiro, Museu Nacional, xvii+717p. (Série Livros 6).

Rodríguez G. 1981. Decapoda; pp. 41-51, in: S.H. Hurlbert, G. Rodríguez and N.D. Santos (eds.). Aquatic Biota of Tropical South America, Part 1: Arthropoda. San Diego: San Diego State University.
Silva, K.C.A., R.A.L. Souza and I.H.A. Cintra. 2002a. Camarão cascudo Macrobrachium amazonicum (Heller, 1862) (Crustacea, Decapoda, Palaemonidae) no Município de Vigia-Pará-Brasil. Boletim TécnicoCientífico do CEPNOR 2(1): 41-73.

Silva, K.C.A., R.A.L. Souza, I.H.A. Cintra and P.A.M. Garrido. 2002b. Camarões capturados em áreas estuarinas no município de VigiaPará-Brasil. Boletim Técnico-Científico do CEPNOR 2(2): 81-96.

Silva, K. C. A., Cintra, I. H. A. and Muniz, A. P. M. 2005. Aspectos bioecológicos de Macrobrachium amazonicum (Heller,1862) a jusante do reservatório da hidroelétrica de Tucuruí - Pará. Boletim Técnico-Científico do CEPNOR 5(1): 55-71.

Silva, M.C.N., F. Lucena-Frédou and J.S. Rosa Filho. 2007. Estudo do crescimento do camarão Macrobrachium amazonicum (Heller, 1862) na ilha de Combú, Belém, estado do Pará. Amazônia: Ciência e Desenvolvimento 2(4): 85-104.

Stimpson, W. 1871. Notes on North American Crustacea in the Museum of the Smithsonian Institution. No. III. Annals of the Lyceum of Natural History of New York 10: 92-136.

Tiefenbacher, L. 1978. Zur Systematik und Verbreitung der Euryrhynchinae (Decapoda, Natantia, Palaemonidae). Crustaceana 35(2): 177-189. (doi: 10.1163/156854078X00088)

Vieira, I.M. 2003. Diversidade de crustáceos das ressacas da Lagoa dos Índios, Tacacá e APA do Curiaú; pp. 53-62, in: L.R.Takiyama and A.Q. Silva (orgs.). Diagnóstico das Ressacas do Estado do Amapá: Bacias do Igarapé da Fortaleza e Rio Curiaú. Macapá. Macapá: CPAQ/IEPA, DGEO/SEMA.

Vieira, I.M. 2008. Inventários rápidos da fauna de crustáceos do Parque Nacional Montanhas do Tumucumaque: Expedições I a V; pp. 66-71, in: F. Bernard (ed.). Inventários Biológicos Rápidos no Parque Nacional Montanhas do Tumucumaque, Amapá, Brasil. RAP Bulletin of Biological Assessment 48. Arlington, VA: Conservation International. (http://www.conservation.org.br/publicacoes/files/ RAP_Tumucumaque.pdf)

Villalobos-Figueiroa, A. 1982. Decapoda; pp. 215-239, in: S.H. Hulbert and A. Villalobos-Figueiroa (eds.). Aquatic Biota of Mexico, Central America and the West Indies. San Diego: San Diego State University.

Wiegmann, A.F.A. 1836. Beschreibung einiger neuen Crustaceen des Berliner Museums aus México umd Brasilien. Archiv für Naturgeschichte 2(1): 145-151.

Wovor, D. and P.K.L. Ng. 2008. Case 3428. Palaemon rosenbergii De Man, 1879 (currently Macrobrachium rosenbergii; Crustacea, Decapoda): proposed conservation of usage by designation of a neotype. Bulletin of Zoological Nomenclature 65(4): 288-293. (http://iczn. org/content/pala emon-rosenbergii-de-man-1879-currentlymacrobrachium-rosenbergii-crustacea-decapoda-prop)

RECEIVED: September 2013

ACCEPTED: April 2014

Published ONLINE: December 2014

EDITORIAL RESPONSIBILITY: Luis Ernesto Arruda Bezerra 